\title{
A reduced basis for a local high definition wind model
}

\author{
J. M. Cascón ${ }^{\mathrm{a}}$, A. Engdahl Y ${ }^{\mathrm{b}}$, L. Ferragut ${ }^{\mathrm{c}}$, E. Hernández ${ }^{\mathrm{d}}$ \\ ${ }^{a}$ Dpto. de Economía e Historia Económica, Universidad de Salamanca, Campus Miguel de Unamuno, Ed. FES, 37007, Salamanca, España, \\ casbar@usal.es \\ ${ }^{b}$ Universidad Técnica Federico Santa María, Avenida España, 1680, Valparaíso, Chile. andrea.engdahl@usm.cl \\ ${ }^{c}$ Inst. Universitario de Física Fundamental y Matemáticas, Universidad de Salamanca, Casas del parque 1, 37008, Salamanca, España, \\ ferragut@usal.es \\ ${ }^{d}$ Universidad Técnica Federico Santa María, Avenida España, 1680, Valparaíso, Chile, erwin.hernandez@usm.cl
}

\begin{abstract}
In this paper we present an application of the reduced basis method to a local high definition adjusted wind model. The model provides a precise description of the wind in 3D and takes into account topography and thermal gradients on the surface by solving only 2D linear equations; the buoyancy forces, slope effects, and mass conservation are also considered. The wind field is adjusted to the point measurements through an optimal control problem in which the wind flux acts as a control on the boundary. In order to use a reduced basis method, we consider an affine decomposition in terms of the parameter related to the friction coefficient and the wind measures at some given observation points. We also design an a posteriori error estimator that is needed to conduct our reduced basis process. Finally, two numerical examples are presented: a test problem and a real-data scenario, we corroborate the correct behavior of the method in both cases.
\end{abstract}

Keywords: Reduced basis method, wind field adjustment, optimal control, A posteriori error estimates

\section{Introduction}

In this paper we implement the reduced basis method for the efficient resolution of an optimal control problem associated to a wind field model. Wind models are a fundamental tool in the study of environmental problems such as dispersion of pollutants, fire propagation, among others. Our starting point is a mass consistent vertical diffusion wind field model. If the significant phenomena that we want to simulate occur in a zone, where the horizontal dimensions are much larger than the vertical one, then an asymptotic approximation of the primitive Navier-Stokes equations can be derived as in the model developed in $[1,2]$. The most salient feature of this asymptotic approach is that it provides a three-dimensional velocity wind field (which satisfies the incompressibility condition in the air layer) governed by a two-dimensional equation, so that it can be coupled with the temperature surface distribution in order to take into account the thermal effects such as sea breezes. In addition, the terrain elevation information is also taken into account by the model.

The validity of this model has the following limits: the nonlinear terms are neglected and it is assumed that the air temperature decreases linearly with the height. On the other hand, the model takes into account buoyancy forces, slope effects, and mass conservation. The wind model presented in this paper is an adaptation of the wind model proposed in [1]. When the data are given by meteorological predictions, an optimal control problem is obtained [2], which can be solved using the adjoint equation-based method. We refer the reader to [3] for the details of this approach. The corresponding numerical approximation leads to linear algebraic systems of equations that are very ill conditioned and quite challenging to solve. In practical applications, the number of equations can be high (roughly between 100 000 and 600 000) and the algebraic system has to be solved many times in the course of a simulation. Moreover, the model requires the estimation of the involved parameters. This adjustment is usually made by genetic algorithm [4], and demands intensive computation. Therefore, the search for efficient solver methods is needed.

The reduced basis method is a procedure for the efficient solution of parametrized partial differential equations. A general formulation of the method and an analysis of their properties can be found in [5] (see also [6]). This method is premised upon a trustworthy technique to approximate the original problem, in our case the finite element 
method (FE). Then, an approximation of the FE solution is computed by means of a Galerkin projection onto a lowdimensional subspace generated by a set of conveniently chosen basis functions, called the reduced basis. The method is composed of two main steps: offline and online. The first one of them requires a considerable computational effort which is carried out only once, and yields the reduced basis system. In the online stage the problem is solved using the basis system generated at the previous step. It must be exceptionally fast. A great amount of applications of the reduced basis method can be found in the literature in different fields (see, for instance, [7, 8, 9]).

The reduced basis methodology is especially appropriate in problems in which real time results are required and when we need to calculate the solution many times, for example when the solution depends on several parameters. In our case, having settled the domain and given that the stations have a particular localization, the solution wind field depends on the point wind measures and on the friction coefficient. Having built the reduced basis system at the offline process, the online process will allow the calculation in real time of the wind field associated to any resulting point measures and to any friction coefficient. This framework can be used, for instance, as an alternative mesoscale-microscale coupling technique for wind resource estimation in local terrains $[10,11,12]$.

The rest of the document is organized as follows: in section 2 the wind model and its discretization are described. Then, in section 3 the reduced basis method and its application to our problem are considered. Finally, in section 4 two numerical examples are reported: a test problem and a real-data scenario.

\section{Wind model.}

In this section we present the wind model. A complete description can be found in $[1,2]$. Our model arrives from an asymptotic analysis of Navier-Stokes equations and gives a three-dimensional convective model governed by a two-dimensional equation. This model adjusts a three-dimensional velocity wind field in a layer under the influence of the orography and temperature distribution.

\subsection{Notation}

Let us consider the three-dimensional domain $\Omega=\{(\mathbf{x}, z) \mid \mathbf{x} \in \omega, H(\mathbf{x})<z<\delta\}$ representing the air layer under study. We assume that the height $\delta$ is small compared to the width, and that the surface height at point $(\mathbf{x}, H(\mathbf{x}))$, is smaller than $\delta$. We decompose the boundary of $\Omega$ into $\partial \Omega=S \cup A \cup L$, where $S=\{(\mathbf{x}, z) \mid \mathbf{x} \in \omega, z=H(\mathbf{x})\}$ is the surface, $A=\{(\mathbf{x}, z) \mid \mathbf{x} \in \omega, z=\delta\}$ is the air upper boundary and $L=\{(\mathbf{x}, z) \mid \mathbf{x} \in \partial \omega, H(\mathbf{x})<z<\delta\}$ is the air lateral boundary; $\omega \subset \mathbb{R}^{2}$ is a two-dimensional normalized bounded domain, representing the projection of the three-dimensional geographical surface $S$. We denote by $(\mathbf{x}, z)$ any point of the three-dimensional domain $\Omega$, and by $\mathbf{x}$ any point of the two-dimensional domain $\omega$.

\subsection{Asymptotic equations}

Consider an air velocity field $\mathbf{U}=(U, V, W)$ and a potential $P$ satisfying the Navier-Stokes equations. Using the fact that the thickness $\delta$ of the considered air layer is small compared with its width, we obtain the following vertical diffusion model:

$$
\begin{aligned}
-\partial_{z z}^{2} \mathbf{V}+\nabla_{\mathbf{x}} P & =0, \\
\partial_{z} P & =\mu T, \\
\nabla_{\mathbf{x}} \cdot \mathbf{V}+\partial_{z} W & =0,
\end{aligned}
$$

where $\mathbf{V}=(U, V)$ denotes the horizontal velocity, $\mu$ is related to buoyancy forces and $T$ is the temperature. We define the horizontal flux at a point $\mathbf{x} \in \omega$ by

$$
\overline{\mathbf{V}}=\int_{H(\mathbf{x})}^{\delta} \mathbf{V}(\mathbf{x}, z) d z
$$

Denoting by $\mathbf{N}$ and $\mathbf{n}$ the inner unit normal vector field to $\partial \Omega$ and to $\partial \omega$, respectively, the boundary conditions can be written as

$$
\begin{array}{rr}
\partial_{z} \mathbf{V}=\zeta \mathbf{V}, \quad(\mathbf{V}, W) \cdot \mathbf{N}=0, & \text { on } S, \\
\partial_{z} \mathbf{V}=0, \quad W=0, & \text { on } A, \\
\overline{\mathbf{V}} \cdot \mathbf{n}=(\delta-H) \mathbf{v}_{m} \cdot \mathbf{n}, & \text { on } \partial \omega .
\end{array}
$$


Here $\mathbf{v}_{m}$ denotes the meteorological wind, which is assumed to be known, horizontal, independent of $z$ and with zero total flux through the lateral boundary, that is,

$$
\partial_{z} \mathbf{v}_{m}=0, \quad \int_{\partial \omega}(\delta-H) \mathbf{v}_{m} \cdot \mathbf{n} d \sigma=0 .
$$

Equations (1) to (6) are well posed: for given $T$ and $\mathbf{v}_{m}$, there exists a unique solution $(\mathbf{V}, W, P$ ) (up to an additive constant for $P$ ). For more details about this convection asymptotic model, see [1]. The solution of problem (1)-(6) can be obtained by explicitly computing $P(\mathbf{x}, z)$ and $\mathbf{V}(\mathbf{x}, z)$ as functions of $z, q(\mathbf{x}), h(\mathbf{x})$ and $\nabla_{\mathbf{x}} p(\mathbf{x})$, where $p$ is a $2 D$ potential.

Equation (1), together with conditions in (4) and (5), yields

$$
\mathbf{V}(\mathbf{x}, z)=m(\mathbf{x}, z) \nabla_{\mathbf{x}} p(\mathbf{x})+k(\mathbf{x}, z) \nabla_{\mathbf{x}} \hat{t}(\mathbf{x})
$$

where

$$
\begin{aligned}
m(\mathbf{x}, z) & =\frac{1}{2} z^{2}-\delta z-\frac{1}{2} H^{2}(\mathbf{x})+(\delta+\xi) H(\mathbf{x})-\xi \delta \\
k(\mathbf{x}, z) & =-\frac{1}{24} z^{4}+\frac{1}{6} \delta z^{3}-\frac{1}{3} \delta^{3} z+\frac{1}{24} H^{4}(\mathbf{x})-\frac{1}{6} H^{3}(\mathbf{x})(\delta+\xi)+\frac{1}{2} \xi \delta H^{2}(\mathbf{x})+\frac{1}{3} \delta^{3} H(\mathbf{x})-\frac{1}{3} \xi \delta^{3},
\end{aligned}
$$

with $\xi=\frac{1}{\zeta}$ being the inverse of the friction coefficient $\zeta$ and $\hat{t}$ being a re-scaled temperature related to the surface temperature $t=t(\mathbf{x})$ by $\hat{t}(\mathbf{x})=\frac{\mu t(\mathbf{x})}{\delta-H(\mathbf{x})}$. We are assuming that the air temperature decreases linearly with the height, $T(\mathbf{x}, z)=t(\mathbf{x}) \frac{\delta-z}{\delta-H(\mathbf{x})}$. The function $p(\mathbf{x})$ is a potential that satisfies the following boundary value problem:

$$
\begin{aligned}
-\nabla_{\mathbf{x}}\left(a \nabla_{\mathbf{x}} p\right) & =\nabla_{\mathbf{x}}\left(r \nabla_{\mathbf{x}} \hat{t}\right) \quad \text { in } \omega \\
a \frac{\partial p}{\partial \mathbf{n}} & =-r \frac{\partial \hat{t}}{\partial \mathbf{n}}+(\delta-H) \mathbf{v}_{m} \cdot \mathbf{n} \quad \text { on } \quad \partial \omega
\end{aligned}
$$

where

and

$$
a=a(\mathbf{x})=\frac{1}{3}(\delta-H(\mathbf{x}))^{2}(3 \xi+\delta-H(\mathbf{x}))
$$

$$
r=r(\mathbf{x})=\frac{1}{30}(\delta-H(\mathbf{x}))^{2}\left(2 \delta^{2}(2 \delta+5 \xi)-2 \delta(\delta-5 \xi) H(\mathbf{x})-(3 \delta+5 \xi) H^{2}(\mathbf{x})+H^{3}(\mathbf{x})\right) .
$$

Summarizing, the solution $\mathbf{V}$ of problem (1)-(6) is obtained by solving the $2 D$ boundary value problem (10) and then $\mathbf{V}$ is explicitly computed using the expression (7).

In practical applications [2], the inverse of the friction coefficient $\xi$ is defined in terms of the roughness of the terrain. Here, we propose a quadratic adjustment:

$$
\xi=d_{0}+d_{1} z_{0}+d_{2} z_{0}^{2}
$$

The parameters $\left\{d_{0}, d_{1}, d_{2}\right\}$ could be computed by a genetic algorithm.

\subsection{Adjustment of point data by solution of an optimal control problem}

In practical applications the wind on the boundary is unknown. Instead, measurements of the wind intensity and direction are given at the points where the weather stations are placed. So we have to reformulate problem (10) so that the given data be the wind velocity at some fixed points.

To simplify the notation, and since in the following we are only concerned with the two-dimensional problem, we omit the subscript ( $)_{\mathbf{x}}$ in the differential operators.

Let $v=(\delta-H) \mathbf{v}_{m} \cdot \mathbf{n}$, then $v \in L_{0}^{2}(\partial \omega)$, where $L_{0}^{2}(\partial \omega)=\left\{v \in L^{2}(\partial \omega) \mid \int_{\partial \omega} v d \sigma=0\right\}$. We are going to reformulate the original problem as an optimal control problem. Given $E$ experimental measurements of the wind velocity $\mathbf{V}_{i}, i=$ $1, \ldots, E$, at $E$ fixed points $P_{i}=\left(\mathbf{x}_{i}, z_{i}\right), i=1, \ldots, E$, we look for the function $v \in L_{0}^{2}(\partial \omega)$ such that the values of $\mathbf{V}\left(\mathbf{x}_{i}, z_{i}\right)$ given by the expression in (7) are as close as possible to the experimental values of $\mathbf{V}_{i}$. Thus, in the optimal control framework we have: 
i) $v \in L_{0}^{2}(\partial \omega)$ is the control;

ii) The state equations are (10);

iii) The regularized cost functional to be minimized is given by

$$
J(v)=\frac{1}{2} \sum_{i=1}^{E} \int_{\omega} \rho_{\varepsilon, i}(\mathbf{x})\left|m\left(\mathbf{x}, z_{i}\right) \nabla p(\mathbf{x})+k\left(\mathbf{x}, z_{i}\right) \nabla \hat{t}(\mathbf{x})-\mathbf{V}_{i}\right|^{2} d \mathbf{x}+\frac{\alpha}{2} \int_{\partial \omega} v^{2} d \sigma,
$$

where $\alpha$ is a regularization parameter and $\rho_{\varepsilon, i}$ is a suitable smoothing function given for example by

$$
\rho_{\varepsilon, i}(\mathbf{x})=\frac{1}{\varepsilon^{2}} \rho\left(\frac{\mathbf{x}-\mathbf{x}_{i}}{\varepsilon}\right), \quad \rho(\mathbf{x})=\left\{\begin{array}{cc}
G \mathrm{e}^{-\frac{1}{1-|\mathbf{x}|^{2}}} & \text { for }|\mathbf{x}|<1 \\
0 & \text { for }|\mathbf{x}| \geq 1
\end{array}\right.
$$

for a small $\varepsilon$, and where $G$ is a constant such that $\int_{\omega} \rho_{\varepsilon, i}(\mathbf{x}) d \mathbf{x}=1$. Here and in the rest of the paper, we denote the Euclidean length of a vector $\mathbf{x}$ by $|\mathbf{x}|$.

With these definitions, the optimal control problem to be solved may be posed as follows:

$$
\begin{gathered}
\text { Find } u \in L_{0}^{2}(\partial \omega) \text { such that: } \\
\qquad \begin{array}{c}
J(u)=\inf _{v \in L_{0}^{2}(\partial \omega)} J(v) .
\end{array}
\end{gathered}
$$

The solution $u$ is characterized by the vanishing of the first variation: $J^{\prime}(u)=0$.

Remark 2.1 (Regularization parameter). The regularization term $\frac{\alpha}{2} \int_{\partial \omega} v^{2} d \sigma$ is necessary for mathematical reasons [3]. In practical applications, we do not usually have a good estimation of the flux at the boundary, and this is the reason why we choose the value of $\alpha$ to be small, typically $\alpha=[0.01,0.1]$.

Remark 2.2 (Alternative regularization term). If we have a good estimation of the flux on the boundary, say $v \approx v^{*}$, then the regularization term can be taken $\frac{\alpha}{2} \int_{\partial \omega}\left(v-v^{*}\right)^{2} d \sigma$, with $\alpha \approx 1$.

\subsection{Weak Formulation}

We here give a characterization of the solution of problem (14) using the adjoint state approach. We refer to [3] for the general theory and [2] for the application to this particular case. We first define the spaces:

$$
\begin{aligned}
& \mathbb{V}:=H^{1}(\omega)=\left\{\varphi \in L^{2}(\omega) \mid \nabla \varphi \in L^{2}(\omega)\right\}, \\
& \mathbb{V}^{\circ}:=\left\{v \in \mathbb{V} \mid \int_{\partial \omega} v d \sigma=0\right\}
\end{aligned}
$$

In order to solve the optimal control problem (14), we derive the following equivalent problem where the unknown $p$ is the potential associated to $u$ and $q$ is its adjoint state (see [2]):

$$
\begin{aligned}
& \text { Find }(p, q) \in \mathbb{V}^{\circ} \times \mathbb{V}^{\circ} \text { such that: } \\
& \int_{\omega} a \nabla p \cdot \nabla \varphi d \mathbf{x}+\frac{1}{\alpha} \int_{\partial \omega} q \varphi d \sigma=-\int_{\omega} r \nabla \hat{t} \cdot \nabla \varphi d \mathbf{x} \quad \forall \varphi \in \mathbb{V}^{\circ}, \\
& \int_{\omega} a \nabla q \cdot \nabla \psi d \mathbf{x}-\sum_{i=1}^{E} \int_{\omega} \rho_{\varepsilon, i} m^{2} \nabla p \cdot \nabla \psi d \mathbf{x}=\sum_{i=1}^{E} \int_{\omega} g_{i} \nabla \psi d \mathbf{x} \quad \forall \psi \in \mathbb{V}^{\circ},
\end{aligned}
$$

where $g_{i}(\mathbf{x}):=\rho_{\varepsilon, i}(\mathbf{x})\left(k \nabla \hat{t}-\mathbf{V}_{i}\right) m\left(\mathbf{x}, z_{i}\right)$. Then, the solution of (14) is given by:

$$
u=-\frac{1}{\alpha} q \quad \text { on } \partial \omega .
$$


Using general control theory (see [3, Chapter1] or [2, Section 3.2]) it can be proved that (14) has a unique solution for $\alpha>0$. This implies the existence and uniqueness of the solution of the previous system. However, we show below that (16)-(17) satisfies an inf-sup condition which reveals that the problem itself is well-posed. We now introduce some notations. Note that the problem above can be equivalently written as:

$$
\begin{aligned}
& \text { Find }(p, q) \in \mathbb{V}^{\circ} \times \mathbb{V}^{\circ} \text { such that: } \\
& \mathcal{A}((p, q),(\varphi, \psi))=\mathcal{F}((\varphi, \psi)) \quad \forall(\varphi, \psi) \in \mathbb{V}^{\circ} \times \mathbb{V}^{\circ},
\end{aligned}
$$

where

$$
\begin{aligned}
\mathcal{A}((p, q),(\varphi, \psi)) & :=\int_{\omega} a \nabla p \cdot \nabla \varphi d \mathbf{x}+\frac{1}{\alpha} \int_{\partial \omega} q \varphi d \sigma+\int_{\omega} a \nabla q \cdot \nabla \psi d \mathbf{x}-\sum_{i=1}^{E} \int_{\omega} \rho_{\varepsilon, i} m^{2} \nabla p \cdot \nabla \psi d \mathbf{x}, \\
\mathcal{F}((\varphi, \psi)) & :=-\int_{\omega} r \nabla \hat{T} \cdot \nabla \varphi d \mathbf{x}+\sum_{i=1}^{E} \int_{\omega} g_{i} \nabla \psi d \mathbf{x} .
\end{aligned}
$$

We denote by $(p, q)_{a}:=\int_{\omega} a \nabla p \nabla q d \mathbf{x}$ the inner product in $\mathbb{V}^{\circ}$, and by $\|p\|_{a}^{2}:=(p, p)_{a}$ its induced norm. Using the trace theorem, there exists a constant $c_{\omega}>0$ such that

$$
\|\varphi\|_{L^{2}(\partial \omega)} \leq c_{\omega}\|\varphi\|_{a} \quad \forall \varphi \in \mathbb{V}^{\circ} .
$$

Finally, because of the definitions of $m, a$, and $\rho_{\epsilon, i}$, there exists positive constants $a_{-}, a_{+}, m_{-}, m_{+}$, and $\rho_{+}$such that

$$
\begin{aligned}
& 0<a_{-} \leq\|a(\mathbf{x})\|_{L^{\infty}(\omega)} \leq a_{+}, \\
& 0<m_{-} \leq\left\|m^{2}(\mathbf{x}, z)\right\|_{L^{\infty}(\Omega)} \leq m_{+}, \\
& \left\|\sum_{i=1}^{E} \rho_{\epsilon}(\mathbf{x})\right\|_{L^{\infty}(\omega)} \leq \rho_{+} .
\end{aligned}
$$

We now prove that the bilinear form $\mathcal{A}(\cdot, \cdot)$ satisfies inf-sup conditions on $\mathbb{V}^{\circ}$. These conditions are equivalent to saying that the linear operator induced by $\mathcal{A}(\cdot, \cdot)$ is bijective, i.e. the problem (19) has a unique solution for any linear $\mathcal{F}(\cdot)[13]$.

Proposition 2.1 (Inf-sup). There exist a constant $\beta_{\mathcal{A}}>0$, which depends on $\frac{\alpha}{c_{\omega}^{2}}$, such that the bilinear form $\mathcal{A}$ satisfies the inf-sup conditions:

$$
\begin{array}{ll}
\sup _{(\varphi, \psi) \in \mathbb{V}^{\circ} \times \mathbb{V}^{\circ}} \frac{\mathcal{A}((p, q),(\varphi, \psi))}{\|(\varphi, \psi)\|_{a}} \geq \beta_{\mathcal{A}}\|(p, q)\|_{a} & \forall(p, q) \in \mathbb{V}^{\circ} \times \mathbb{V}^{\circ}, \\
\sup _{(p, q) \in \mathbb{V}^{\circ} \times \mathbb{V}^{\circ}} \frac{\mathcal{A}((p, q),(\varphi, \psi))}{\|(p, q)\|_{a}} \geq \beta_{\mathcal{H}}\|(\varphi, \psi)\|_{a} & \forall(\varphi, \psi) \in \mathbb{V}^{\circ} \times \mathbb{V}^{\circ},
\end{array}
$$

where $\|(p, q)\|_{a}=\left(\|p\|_{a}^{2}+\|q\|_{a}^{2}\right)^{1 / 2}$. In fact, we obtain

$$
\beta_{\mathcal{A}} \geq\left[2 \sqrt{1+\max \left\{\frac{c_{\omega}^{2}}{2 \alpha}, \frac{\rho_{+} m_{+}}{2 a_{-}}\right\}^{2}}\right]^{-1} .
$$

Proof. We prove only (24); estimate (25) can be derived similarly. On one hand, combining Young's inequality with the parameter $\epsilon_{1}>0$ and estimation (22), we derive

$$
\int_{\partial \omega} p q d \sigma \geq-\frac{\epsilon_{1}}{2}\|p\|_{L^{2}(\partial \omega)}^{2}-\frac{1}{2 \epsilon_{1}}\|q\|_{L^{2}(\partial \omega)}^{2} \geq-\frac{\epsilon_{1} c_{\omega}^{2}}{2}\|p\|_{L^{2}(\omega)}^{2}-\frac{1}{2 \epsilon_{1}}\|q\|_{L^{2}(\partial \omega)}^{2} .
$$

On the other hand, again using Young's inequality with the parameter $\epsilon_{2}>0$ and the bounds for functions $a, m$ and $\rho_{\epsilon, i}$ in (23) we infer

$$
-\sum_{i=1}^{E} \int_{\omega} \rho_{\varepsilon, i} m^{2} \nabla p \cdot \nabla q d \mathbf{x} \geq-\frac{1}{2 \epsilon_{2}} \sum_{i=1}^{E} \int_{\omega} \rho_{\varepsilon, i} m^{2} \nabla p \cdot \nabla p d \mathbf{x}-\frac{\epsilon_{2}}{2} \frac{\rho_{+} m_{+}}{a_{-}}\|q\|_{a}^{2} .
$$


We now choose $\varphi=p+M q$ and $\psi=q-M p$ where $M \in \mathbb{R}$ is a parameter to be found later. By definition of the bilinear form $\mathcal{A}(\cdot, \cdot)$ and the equations (27)-(28), we have

$$
\begin{aligned}
\mathcal{A}((p, q),(\varphi, \psi))= & \|p\|_{a}^{2}+\frac{M}{\alpha}\|q\|_{L^{2}(\partial \omega)}^{2}+\frac{1}{\alpha} \int_{\partial \omega} p q d \sigma+\|q\|_{a}^{2} \\
& -\sum_{i=1}^{E} \int_{\omega} \rho_{\epsilon, i} m^{2} \nabla p \cdot \nabla q d \mathbf{x}+M \sum_{i=1}^{E} \int_{\omega} \rho_{\epsilon, i} m^{2} \nabla p \cdot \nabla p d \mathbf{x} \\
\geq & \left(1-\frac{c_{\omega}^{2}}{2 \alpha} \epsilon_{1}\right)\|p\|_{a}^{2}+\left(M-\frac{1}{2 \epsilon_{1}}\right) \frac{1}{\alpha}\|q\|_{L^{2}(\partial \omega)}^{2} \\
& +\left(1-\epsilon_{2} \frac{\rho_{+}}{2} \frac{m_{+}}{a_{-}}\right)\|q\|_{a}^{2}+\left(M-\frac{1}{2 \epsilon_{2}}\right) \sum_{i=1}^{E} \int_{\omega} \rho_{\varepsilon, i} m^{2} \nabla p \cdot \nabla p d \mathbf{x} .
\end{aligned}
$$

We then fix the parameters $\epsilon_{1}, \epsilon_{2}$ and $M$ to be:

$$
\epsilon_{1}=\frac{\alpha}{c_{\omega}^{2}}, \quad \epsilon_{2}=\frac{a_{-}}{\rho_{+} m_{+}}, \quad M \geq \max \left\{\frac{1}{2 \epsilon_{1}}, \frac{1}{2 \epsilon_{2}}\right\},
$$

to obtain

$$
\mathcal{A}((p, q),(\varphi, \psi)) \geq \frac{1}{2}\left(\|p\|_{a}^{2}+\|q\|_{a}^{2}\right)
$$

Finally, from the fact that

$$
\sup _{(\varphi, \psi) \in \mathbb{V}^{\circ} \times \mathbb{V}^{\circ}} \frac{\mathcal{A}((p, q),(\varphi, \psi))}{\|(\varphi, \psi)\|_{a}} \geq \frac{\frac{1}{2}\left(\|p\|_{a}^{2}+\|q\|_{a}^{2}\right)}{\sqrt{1+M^{2}}\|(p, q)\|_{a}} \geq \frac{\|(p, q)\|_{a}^{2}}{2 \sqrt{1+M^{2}}\|(p, q)\|_{\mathbb{V}^{\circ} \times \mathbb{V}^{\circ}}}
$$

we conclude the desired result.

Remark 2.3 (Discrete inf-sup conditons). We note that the proof of Proposition 2.1 does not require any characteristic of the space $\mathbb{V}^{\circ} \times \mathbb{V}^{\circ}$, and therefore the result is also valid at the discrete level, that is, the problem (19) has a unique solution for any finite dimensional subspace of $\mathbb{V}^{\circ}$.

\subsection{Finite Element Approximation}

A reduced basis method hinges on a procedure that is able to provide a trustworthy approximation, in our case, the finite element method (FE) on a finer mesh. To obtain the FE approximation of (19), we first consider a shape regular triangulation $\mathcal{T}_{h}:=\{T\}$ of $\omega$ where $h$ denotes its size (i.e. $h:=\max _{T \in \mathcal{T}} \operatorname{diam}(T)$ ). We then introduce $\mathbb{V}_{h}^{\circ} \subset \mathbb{V}^{\circ}$ as the space of continuous, piecewise linear functions subordinated to $\mathcal{T}_{h}$. Recall that a function $\varphi_{h} \in \mathbb{V}_{h}^{\circ}$ has zero mean value on $\partial \omega$, i.e. $\int_{\partial \omega} \varphi_{h}=0$. Finally, the corresponding FE approximation of (19) reads as follows:

$$
\begin{aligned}
& \text { Find }\left(p_{h}, q_{h}\right) \in \mathbb{V}_{h}^{\circ} \times \mathbb{V}_{h}^{\circ} \text { such that: } \\
& \mathcal{A}\left(\left(p_{h}, q_{h}\right),\left(\varphi_{h}, \psi_{h}\right)\right)=\mathcal{F}\left(\left(\varphi_{h}, \psi_{h}\right)\right), \quad \forall\left(\varphi_{h}, \psi_{h}\right) \in \mathbb{V}_{h}^{\circ} \times \mathbb{V}_{h}^{\circ}
\end{aligned}
$$

We note that the previous problem is well-posed and has an unique solution, (see Remark 2.3). Hereafter, and following [6], we will refer to the FE approximation as the "truth" FE solution.

\section{Reduced Basis Approximation}

In this section we design a reduced basis procedure to approximate the "truth" FE solution with minimal computational effort. In order to make the article self-contained we first present briefly the RB method (for details see [5]). 
Let $\mathbb{X}$ be a Hilbert space with inner product $(\cdot, \cdot)_{\mathbb{X}}$ and associated norm $\|\cdot\|_{\mathbb{X}}$, we consider the following general model problem:

Find $u(\gamma) \in \mathbb{X}$ such that:

$a(u(\mu), v ; \gamma)=l(v ; \gamma) \quad \forall v \in \mathbb{X}$,

where $a(., . ; \gamma)$ is a continuous bilinear form satisfying an inf-sup condition and $l(. ; \gamma)$ is a continuous linear functional. Both depend on a set of parameters $\gamma \in \mathcal{D} \subset \mathbb{R}^{p}$; where $\mathcal{D}$ denotes the domain of variability of the parameters. We also introduce a norm $\|\cdot\|_{\mathbb{X}(\gamma)}$, equivalent to the norm $\|\cdot\|_{\mathbb{X}}$ of $\mathbb{X}$, but $\gamma$ dependent. The RB method requires affine parameter dependence of the bilinear form $a(.$, .; .) and of the functional $l(\cdot ;$.$) , that is, we assume the following representation:$

$$
\begin{aligned}
a(u, v ; \gamma)=\sum_{l=1}^{J_{a}} \Theta_{a}^{l}(\gamma) a^{l}(u, v) & \forall u, v \in \mathbb{X}, \forall \gamma \in \mathcal{D}, \\
l(v ; \gamma)=\sum_{l=1}^{J_{l}} \Theta_{l}^{l}(\gamma) l^{l}(v) & \forall v \in \mathbb{X}, \forall \gamma \in \mathcal{D},
\end{aligned}
$$

where $\Theta_{a}^{l}(\cdot), \Theta_{l}^{l}(\cdot): \mathcal{D} \rightarrow \mathbb{R}$ are functions of $\gamma$ while $a^{l}(\cdot, \cdot)$ and $l^{l}(\cdot)$ are parameter independent.

We also assume that the problem (30) is approximated with a reliable and high cost numerical procedure, for example a FE method on a finer mesh. If $\mathbb{X}_{h} \subset \mathbb{X}$ is a finite dimensional subspace, we introduce $u_{h}(\gamma) \in \mathbb{X}_{h}$ as the solution of the discrete problem:

$$
a\left(u_{h}(\gamma), v_{h} ; \gamma\right)=l\left(v_{h} ; \gamma\right) \quad \forall v_{h} \in \mathbb{X}_{h}
$$

The aim of the RB method is to approximate the FE solution for any parameter $\gamma \in \mathcal{D}$ with minimal computational effort. In order to do that, the method selects a $N$-dimensional subspace $\mathbb{X}_{N} \subset \mathbb{X}_{h}$ spanned by the set $\left\{\zeta_{1}, \ldots, \zeta_{N}\right\}$ of basis functions, where $N$ is small enough compared with the dimension of $\mathbb{X}_{h}$. Then, the reduced basis approximation $u_{N}(\gamma) \in \mathbb{X}_{N}$ is introduced as the Galerkin projection onto the low dimensional subspace $\mathbb{X}_{N}$ :

$$
a\left(u_{N}(\gamma), v_{N} ; \gamma\right)=l\left(v_{N} ; \gamma\right) \quad \forall v_{N} \in \mathbb{X}_{N}
$$

One of the key ingredients when the method selects the basis function of $\mathbb{X}_{N}$ is a sharp and inexpensive a posteriori error bound, $\Delta_{N}(\gamma)$, of the error $e(\gamma)=u_{h}(\gamma)-u_{N}(\gamma)$. The estimator, $\Delta_{N}(\gamma)$ usually depends on the residual of (31). Note, that given any approximation $u_{N}(\gamma)$ of the solution $u_{h}(\gamma)$ of the problem (31), the error $e(\gamma)=u_{h}(\gamma)-u_{N}(\gamma) \in \mathbb{X}_{h}$ satisfies

$$
a\left(e(\mu), v_{h} ; \gamma\right)=\left(r\left(u_{N}(\gamma)\right), v_{h}\right)_{\mathbb{X}}
$$

where $r\left(u_{N}(\gamma)\right) \in \mathbb{X}_{h}$ denotes the Riesz representation of the residual and is given by

$$
\left(r\left(u_{N}(\gamma)\right), v_{h}\right)_{\mathbb{X}}=l\left(v_{h} ; \gamma\right)-a\left(u_{N}(\mu), v_{h} ; \gamma\right) \quad \forall v_{h} \in \mathbb{X}_{h}
$$

This implies

$$
\|e(\gamma)\|_{\mathbb{X}(\gamma)} \preccurlyeq\left\|r\left(u_{N}(\gamma)\right)\right\|_{\mathbb{X}}:=\sup _{v_{h} \in \mathbb{X}_{h}} \frac{\left|l\left(v_{h} ; \gamma\right)-a\left(u_{N}(\gamma), v_{h} ; \gamma\right)\right|}{\left\|v_{h}\right\|_{\mathbb{X}}} \preccurlyeq\|e(\gamma)\|_{\mathbb{X}(\gamma)},
$$

where the hidden constants depend on inf-sup and continuinity constants, and on the parameter $\gamma$.

We can now describe the two main steps of the RB method:

- Offline Process. The RB method builds a sequence of approximation spaces $\mathbb{X}_{1} \subset \mathbb{X}_{2} \subset \ldots, \subset \mathbb{X}_{N} \subset \mathbb{X}_{h}$ step by step so that at the end of the process it gets a set of "optimal" functions $\left\{\zeta_{1}, \ldots, \zeta_{N}\right\}$ spanning the space $\mathbb{X}_{N}$. The construction of the sequence of approximation spaces is based on a greedy algorithm. First it is necessary to discretize the parameters in $\mathcal{D}$. We call $\Xi$ the discrete set of parameters. Having chosen a random parameter $\gamma_{1}$, the problem (31) is solved, this solution is the first element of the basis $\zeta_{1} \in \mathbb{X}_{N}$. Suppose now that the first $k$ elements $\mathbb{X}_{k}$ have already been obtained. Then, for each $\gamma \in \Xi$ we obtain the approximate solution $u_{k}(\mu)$ of the problem (32) as the Galerkin projection onto the subspace generated by $\mathbb{X}_{k}=\left\langle\zeta_{1}, \ldots, \zeta_{k}\right\rangle$ associated to the set of parameters $S_{k}=\left\{\gamma_{1}, \ldots, \gamma_{k}\right\}$. At this point we select the parameter associated to the solution $u_{k}(\gamma)$ with the highest estimator, i.e.:

$$
\gamma_{k+1}=\arg \max _{\gamma \in \Xi} \Delta_{k}(\gamma)
$$


Finally the new element of the basis $\zeta_{k+1}$ is chosen as the solution of the problem (31) with parameter $\gamma=\gamma_{k+1}$. The cardinality of the basis, $N$, can be set directly, or through a prescribed tolerance over the estimator. The affine decomposition makes its calculation independent of the dimension of the high-fidelity model. However the cardinality of $\Xi$ still play a major role since the estimator has to be computed for all the parameter points in $\Xi$. The set of functions is usually orthogonalized.

- Online process. Given a parameter $\gamma \in \mathcal{D}$, the problem (32) is solved to obtain $u_{N}(\gamma)$. The associated system has a smaller dimension when compared to the original, even though the associated matrix does not have a sparse structure.

In our case, $\mathbb{X}$ is the space $\mathbb{V}^{\circ} \times \mathbb{V}^{\circ}$, and the unknown is $u=(p, q)$. The set of parameters includes the wind measures at the stations $\left\{\mathbf{V}_{i}=\left(V_{i}^{1}, V_{i}^{2}\right)^{T}\right\}_{i=1}^{E}$ and the coefficient $\mathbf{d}:=\left\{d_{0}, d_{1}, d_{2}\right\}$ involved in the adjustment of the inverse of the friction coefficient $\xi(12)$, that is:

$$
\gamma \equiv\left\{\mathbf{d},\left\{\mathbf{V}_{i}\right\}_{i=1}^{E}\right\}=\left\{d_{0}, d_{1}, d_{2},\left\{\left(V_{i}^{1}, V_{i}^{2}\right)^{T}\right\}_{i=1}^{E}\right\}
$$

The bilinear form $a(\cdot, \cdot ; \gamma)$ and linear functional $l(\cdot ; \gamma)$ are given by $\mathcal{A}(\cdot, \cdot ; \mathbf{d})$ and $\mathcal{F}(\cdot ; \gamma)$ defined in $(20)$ and $(21)$, respectively. To make explicit this dependence, we write from now, $\mathcal{A}_{\gamma}(\cdot, \cdot)$ and $\mathcal{F}_{\gamma}(\cdot)$. In order to have a parameter independent norm, we first need to fix mean values $\left\{\bar{d}_{0}, \bar{d}_{1}, \bar{d}_{2}\right\}$ of parameters in the expansion of $\xi$ and then we introduce the inner product and its associated norm as:

$$
(p, q)_{\bar{a}}:=\int_{\omega} \bar{a} \nabla p \nabla q d \mathbf{x}, \quad\|p\|_{\bar{a}}:=\sqrt{(p, p)_{\bar{a}}}, \quad \forall, p, q \in \mathbb{V}^{\circ},
$$

where $\bar{a}=a\left(\bar{d}_{0}, \bar{d}_{1}, \bar{d}_{2}\right)$. That is, in our framework $\|\cdot\|_{\mathbb{X}}:=\|(\cdot, \cdot)\|_{\bar{a}}$ and $\|\cdot\| \mathbb{X}(\gamma):=\|(\cdot, \cdot)\|_{a}$.

\subsection{Affine Decomposition}

We now show that the problem (19) admits an affine decomposition, therefore its resolution can be approached by means of the reduced basis method.

By simplicity, in the following we assume uniform temperature on the surface so that the temperature gradient $\nabla \hat{T}=0$.

As we commented before, we consider as parameters the coefficients $\left\{d_{0}, d_{1}, d_{2}\right\}$ of the expansion of the inverse of the friction coefficient $\xi(12)$ and the wind measures at the observation points $\left\{\mathbf{V}_{i}=\left(V_{i}^{1}, V_{i}^{2}\right)^{T}\right\}_{i=1}^{E}$, (36). We recall that the coefficients $a, m$ and $k$ in (11)-(8))-(9) depend on the parameter $\xi$ (see [2]). By expanding the terms in $\xi$, and using (12) we rewrite the coefficients $a, m$ and $k$ as functions of the roughness of the terrain, $z_{0}$ :

$$
\begin{aligned}
a & =\frac{1}{3}(\delta-H)^{3}+(H-\delta)^{2} \xi=a_{0}+a_{1} \xi=a_{0}+a_{1}\left(d_{0}+d_{1} z_{0}+d_{2} z_{0}^{2}\right) \\
m & =-\frac{1}{2}(H-z)(H+z-2 \delta)+(H-\delta) \xi=m_{0}+m_{1} \xi=m_{0}+m_{1}\left(d_{0}+d_{1} z_{0}+d_{2} z_{0}^{2}\right) \\
k & =\left(-\frac{1}{24} z^{4}+\frac{1}{6} \delta z^{3}-\frac{1}{3} \delta^{3} z+\frac{1}{24} H^{4}-\frac{1}{6} \delta H^{3}+\frac{1}{3} \delta^{3} H\right),+\left(-\frac{1}{6} H^{3}+\frac{1}{2} \delta H^{2}-\frac{1}{3} \delta^{3}\right) \xi=k_{0}+k_{1}\left(d_{0}+d_{1} z_{0}+d_{2} z_{0}^{2}\right)
\end{aligned}
$$

As a consequence, the problem (19) admits an affine decomposition in terms of the set of parameters $\gamma$ (36). We write below the explicit decomposition for the system associated to the discrete problem (29). Given a finite element basis $\left\{\phi_{j}\right\}_{j=1}^{\mathcal{N}}$ of $\mathbb{V}_{h}^{\circ}$, the discretization of the problem (29) provides the system

$$
A_{\gamma}\left[\begin{array}{l}
\mathbf{p} \\
\mathbf{q}
\end{array}\right]=\mathbf{F}_{\gamma}
$$


where, the matrix system $A_{\gamma}$ admits the following decomposition:

$$
\begin{aligned}
A_{\gamma}= & A_{0}+\sum_{l=1}^{9} \theta_{A}^{l}\left(d_{0}, d_{1}, d_{2}\right) A_{l} \\
= & {\left[\begin{array}{cc}
B_{0} & C \\
D_{0} & B_{0}
\end{array}\right]+d_{0}\left[\begin{array}{cc}
B_{1} & 0 \\
D_{1} & B_{1}
\end{array}\right]+d_{1}\left[\begin{array}{cc}
B_{2} & 0 \\
D_{2} & B_{2}
\end{array}\right]+d_{2}\left[\begin{array}{cc}
B_{3} & 0 \\
D_{3} & B_{3}
\end{array}\right]+d_{0} d_{1}\left[\begin{array}{cc}
0 & 0 \\
D_{4} & 0
\end{array}\right]+d_{0} d_{2}\left[\begin{array}{cc}
0 & 0 \\
D_{5} & 0
\end{array}\right] } \\
& +d_{1} d_{2}\left[\begin{array}{cc}
0 & 0 \\
D_{6} & 0
\end{array}\right]+d_{0}^{2}\left[\begin{array}{cc}
0 & 0 \\
D_{7} & 0
\end{array}\right]+d_{1}^{2}\left[\begin{array}{cc}
0 & 0 \\
D_{8} & 0
\end{array}\right]+d_{2}^{2}\left[\begin{array}{cc}
0 & 0 \\
D_{9} & 0
\end{array}\right]
\end{aligned}
$$

with

$$
\begin{array}{rlrl}
B_{0} & =\left\{\int_{\omega} a_{0} \nabla \phi_{j} \cdot \nabla \phi_{r}\right\}_{j, r} & B_{1} & =\left\{\int_{\omega} a_{1} \nabla \phi_{j} \cdot \nabla \phi_{r}\right\}_{j, r} \\
B_{2} & =\left\{\int_{\omega} a_{1} z_{0} \nabla \phi_{j} \cdot \nabla \phi_{r}\right\}_{j, r} & B_{3} & =\left\{\int_{\omega} a_{1} z_{0}^{2} \nabla \phi_{j} \cdot \nabla \phi_{r}\right\}_{j, r} \\
D_{0} & =-\left\{\sum_{i=1}^{E} \int_{\omega} \rho_{\varepsilon, i} m_{0}^{2} \nabla \phi_{j} \cdot \nabla \phi_{r}\right\}_{j, r} & D_{1} & =-2\left\{\sum_{i=1}^{E} \int_{\omega} \rho_{\varepsilon, i} m_{0} m_{1} \nabla \phi_{j} \cdot \nabla \phi_{r}\right\}_{j, r} \\
D_{2} & =-2\left\{\sum_{i=1}^{E} \int_{\omega} \rho_{\varepsilon, i} m_{0} m_{1} z_{0} \nabla \phi_{j} \cdot \nabla \phi_{r}\right\}_{j, r} & D_{3} & =-2\left\{\sum_{i=1}^{E} \int_{\omega} \rho_{\varepsilon, i} m_{0} m_{1} z_{0}^{2} \nabla \phi_{j} \cdot \nabla \phi_{r}\right\}_{j, r} \\
D_{4} & =-2\left\{\sum_{i=1}^{E} \int_{\omega} \rho_{\varepsilon, i} m_{1}^{2} z_{0} \nabla \phi_{j} \cdot \nabla \phi_{r}\right\}_{j, r} & D_{5} & =-2\left\{\sum_{i=1}^{E} \int_{\omega} \rho_{\varepsilon, i} m_{1}^{2} z_{0}^{2} \nabla \phi_{j} \cdot \nabla \phi_{r}\right\}_{j, r} \\
D_{6} & =-2\left\{\sum_{i=1}^{E} \int_{\omega} \rho_{\varepsilon, i} m_{1}^{2} z_{0}^{3} \nabla \phi_{j} \cdot \nabla \phi_{r}\right\}_{j, r} & D_{7}=-\left\{\sum_{i=1}^{E} \int_{\omega} \rho_{\varepsilon, i} m_{1}^{2} \nabla \phi_{j} \cdot \nabla \phi_{r}\right\}_{j, r} \\
D_{8}=-\left\{\sum_{i=1}^{E} \int_{\omega} \rho_{\varepsilon, i} m_{1}^{2} z_{0}^{2} \nabla \phi_{j} \cdot \nabla \phi_{r}\right\}_{j, r} & D_{9}=-\left\{\sum_{i=1}^{E} \int_{\omega} \rho_{\varepsilon, i} m_{1}^{2} z_{0}^{4} \nabla \phi_{j} \cdot \nabla \phi_{r}\right\}_{j, r},
\end{array}
$$

and $1 \leq j, r \leq \mathcal{N}$. On the other hand, the right hand side vector $\mathbf{F}$ can be written as:

$$
\mathbf{F}_{\gamma}=-\sum_{i=1}^{E}\left\{\mathbf{V}_{i}\left[\begin{array}{c}
0 \\
\mathbf{F}_{i}^{0}
\end{array}\right]+d_{0} \mathbf{V}_{i}\left[\begin{array}{c}
0 \\
\mathbf{F}_{i}^{1}
\end{array}\right]+d_{1} \mathbf{V}_{i}\left[\begin{array}{c}
0 \\
\mathbf{F}_{i}^{2}
\end{array}\right]+d_{2} \mathbf{V}_{i}\left[\begin{array}{c}
0 \\
\mathbf{F}_{i}^{3}
\end{array}\right]\right\},
$$

where the parameter independent vectors $\mathbf{F}_{i}^{l}$ for $l=0,1,2,3$ and $i=1, \ldots, E$ are given by

$$
\begin{array}{rlrl}
\mathbf{F}_{i}^{0} & =\left\{\int_{\omega} \rho_{\varepsilon, i} m_{0} \nabla \phi_{j}\right\}_{j} & \mathbf{F}_{i}^{1} & =\left\{\int_{\omega} \rho_{\varepsilon, i} m_{1} \nabla \phi_{j}\right\}_{j} \\
\mathbf{F}_{i}^{2} & =\left\{\int_{\omega} \rho_{\varepsilon, i} m_{1} z_{0} \nabla \phi_{j}\right\}_{j} & \mathbf{F}_{i}^{3}=\left\{\int_{\omega} \rho_{\varepsilon, i} m_{1} z_{0}^{2} \nabla \phi_{j}\right\}_{j}
\end{array}
$$

with $1 \leq j \leq \mathcal{N}$.

\subsection{Definition of Reduced Spaces and Well-Posedness of Reduced Problem.}

We select the same reduced space for the state and its adjoint, i.e. $\mathbb{X}_{N}:=\mathbb{V}^{\circ}{ }_{N} \times \mathbb{V}^{\circ}{ }_{N}$, with $\mathbb{V}^{\circ}{ }_{N} \subset \mathbb{V}_{h}^{\circ}$. This particular choice guarantees the existence and uniqueness of the solution of the reduced problem (32), because Remark 2.3 also applies to the reduced level. Another selection can improve the error estimation, however requires an ad-hoc analysis (see [14]). 


\subsection{A posteriori Error Bound}

As we have mentioned before, an essential step for the method is the a posteriori error bound, it allows us to enable the offline process. Note that the bound that we seek (34), is a stability condition for the error in terms of the residual.

We now introduce some notations. Let $e_{p}(\gamma):=p_{h}-p_{N}$ and $e_{q}(\gamma):=q_{h}-q_{N}$ be the errors associated to the RB approximations, that satisfy:

$$
\mathcal{A}_{\gamma}\left(\left(e_{p}(\gamma), e_{q}(\gamma)\right),\left(\varphi_{h}, \psi_{h}\right)\right)=\mathcal{R}_{\gamma}\left(\left(\varphi_{h}, \psi_{h}\right)\right), \quad \forall\left(\varphi_{h}, \psi_{h}\right) \in \mathbb{V}_{h}^{\circ} \times \mathbb{V}_{h}^{\circ}
$$

The previous equation arises by subtracting the FE problem (31) and its RB counterpart (32), and where the forms $a(\cdot, \cdot)$ and $l(\cdot)$ are replaced by $\mathcal{A}_{\gamma}(\cdot, \cdot)$ and $\mathcal{F}_{\gamma}(\cdot)$, respectively. The corresponding residual $\mathcal{R}_{\gamma}\left(\varphi_{h}, \phi_{h}\right)$ is

$$
\begin{aligned}
\mathcal{R}_{\gamma}\left(\left(\varphi_{h}, \psi_{h}\right)\right)= & \mathcal{F}_{\gamma}\left(\varphi_{h}, \psi_{h}\right)-\mathcal{A}_{\gamma}\left(\left(p_{N}, q_{N}\right),\left(\varphi_{h}, \psi_{h}\right)\right) \\
= & \sum_{i=1}^{E} \int_{\omega} g_{i} \nabla \psi_{h} d \mathbf{x}-\int_{\omega} a \nabla p_{N} \nabla \varphi d \mathbf{x}-\frac{1}{\alpha} \int_{\partial \omega} q_{N} \varphi d \\
& -\int_{\omega} a \nabla q_{N} \nabla \psi d \mathbf{x}+\sum_{i=1}^{E} \int_{\omega} \rho_{\epsilon, i}\left(m \nabla p_{N}\right)(m \nabla \psi) d \mathbf{x} \\
= & : R_{\gamma, 1}\left(\varphi_{h}\right)+R_{\gamma, 2}\left(\psi_{h}\right) .
\end{aligned}
$$

Finally, we introduce the Riesz representation $r_{\gamma, 1} \in \mathbb{V}_{h}^{\circ}\left(\right.$ resp. $\left.r_{\gamma, 2} \in \mathbb{V}_{h}^{\circ}\right)$ of $R_{\gamma, 1}(\cdot)\left(\right.$ resp. $\left.R_{\gamma, 2}(\cdot)\right)$ as:

$$
\left(r_{\gamma, 1}, \varphi_{h}\right)_{\bar{a}}=R_{\gamma, 1}\left(\varphi_{h}\right), \quad\left(r_{\gamma, 2}, \varphi_{h}\right)_{\bar{a}}=R_{\gamma, 2}\left(\varphi_{h}\right), \quad \forall \varphi_{h} \in \mathbb{V}_{h}^{\circ}
$$

The following result give us the bound that will be used in the offline process, to obtain efficient computable error estimates.

Proposition 3.1. Let $e_{p}(\gamma):=p_{h}-p_{N}$ and $e_{q}(\gamma):=q_{h}-q_{N}$ be the errors associated to the RB approximations. Then, there exist constants $C_{1}, C_{2} \geq 0$ such that

$$
\left\|e_{p}(\gamma)\right\|_{a}+\left\|e_{q}(\gamma)\right\|_{a} \leq C_{1}\left\|r_{\gamma, 1}\right\|_{\bar{a}}+C_{2}\left\|r_{\gamma, 2}\right\|_{\bar{a}}
$$

where

$$
C_{1}=\sqrt{\frac{a_{+}}{a_{-}}}\left(1+\frac{\rho_{+} m_{+}}{a_{-}}+c_{\omega} \sqrt{\frac{\rho_{+} m_{+}}{\alpha a_{-}}}\right), \quad C_{2}=\sqrt{\frac{a_{+}}{a_{-}}}\left(1+\frac{c_{\omega}^{2}}{\alpha}+c_{\omega} \sqrt{\frac{\rho_{+} m_{+}}{\alpha a_{-}}}\right) .
$$

Proof. For simplicity, we omit the subscript $h$ on functions in this proof. Let $\left(p_{1}, q_{1}\right),\left(p_{2}, q_{2}\right)$ in $\mathbb{V}_{h}^{\circ} \times \mathbb{V}_{h}^{\circ}$, be the solutions of the following problems, respectively,

$$
\begin{aligned}
\int_{\omega} a \nabla p_{1} \nabla \varphi d \mathbf{x}+\frac{1}{\alpha} \int_{\partial \omega} q_{1} \varphi d \sigma & =0 \quad \forall \varphi \in \mathbb{V}_{h}^{\circ}, \\
\int_{\omega} a \nabla q_{1} \nabla \psi d \mathbf{x}-\sum_{i=1}^{E} \int_{\omega} \varphi_{\epsilon, i}\left(m \nabla p_{1}\right)(m \nabla \psi) d \mathbf{x} & =\left(r_{\gamma, 2}, \psi\right)_{\bar{a}} \quad \forall \psi \in \mathbb{V}_{h}^{\circ},
\end{aligned}
$$

and

$$
\begin{aligned}
\int_{\omega} a \nabla p_{2} \nabla \varphi d \mathbf{x}+\frac{1}{\alpha} \int_{\partial \omega} q_{2} \varphi d \sigma & =\left(r_{\gamma, 1}, \varphi\right)_{\bar{a}} \quad \forall \varphi \in \mathbb{V}_{h}^{\circ}, \\
\int_{\omega} a \nabla q_{2} \nabla \psi d \mathbf{x}-\sum_{i=1}^{E} \int_{\omega} \rho_{\epsilon, i}\left(m \nabla p_{2}\right)(m \nabla \psi) d \mathbf{x} & =0 \quad \forall \psi \in \mathbb{V}_{h}^{\circ},
\end{aligned}
$$

Note that, according to Proposition 2.1, the problems (43)-(44) and (45)-(46) have unique solutions. Therefore, by linearity, we can split the error functions as

$$
e_{p}(\gamma)=p_{1}+p_{2} \quad \text { and } \quad e_{q}(\gamma)=q_{1}+q_{2}
$$


We estimate each term separately. First, taking $\varphi=p_{1}$ in (43), we have

$$
\left\|p_{1}\right\|_{a} \leq \frac{c_{\omega}}{\alpha}\left\|q_{1}\right\|_{L^{2}(\partial \omega)}
$$

On the other hand, replacing $\varphi=q_{1}$ in (43) and $\psi=p_{1}$ in (44), and subtracting, we get

$$
\frac{1}{\alpha} \int_{\partial \omega} q_{1}^{2} d \sigma+\sum_{i=1}^{E} \int_{\omega} \rho_{\epsilon, i}\left(m \nabla p_{1}\right)^{2} d \mathbf{x}=-\left(r_{\gamma, 2}, p_{1}\right)_{\bar{a}}
$$

then

$$
\frac{1}{\alpha}\left\|q_{1}\right\|_{L^{2}(\partial \omega)}^{2} \leq\left\|r_{\gamma, 2}\right\|_{\bar{a}}\left\|p_{1}\right\|_{\bar{a}} \leq \sqrt{\frac{a_{+}}{a_{-}}}\left\|r_{\gamma, 2}\right\|_{\bar{a}}\left\|p_{1}\right\|_{a}
$$

and, from (47), we arrive at

$$
\left\|p_{1}\right\|_{\bar{a}}^{2} \leq \frac{c_{\omega}^{2}}{\alpha^{2}}\left\|q_{1}\right\|_{L^{2}(\partial \omega)}^{2} \leq \frac{c_{\omega}^{2}}{\alpha} \sqrt{\frac{a_{+}}{a_{-}}}\left\|r_{\gamma, 2}\right\|_{\bar{a}}\left\|p_{1}\right\|_{a}
$$

and hence

$$
\left\|p_{1}\right\|_{a} \leq \frac{c_{\omega}^{2}}{\alpha} \sqrt{\frac{a_{+}}{a_{-}}}\left\|r_{\gamma, 2}\right\|_{\bar{a}}
$$

We now proceed with the term $q_{1}$. By taking $\psi=q_{1}$ in (44), we obtain

$$
\begin{aligned}
\left\|q_{1}\right\|_{a}^{2} & =\sum_{i=1}^{E} \int_{\omega} \rho_{\epsilon, i}\left(m \nabla p_{1}\right)\left(m \nabla q_{1}\right) d \mathbf{x}+\left(r_{\gamma, 2}, q_{1}\right)_{\bar{a}} \\
& \leq\left(\sum_{i=1}^{E} \int_{\omega} \varphi_{\epsilon, i} m^{2}\left|\nabla p_{1}\right|^{2} d \mathbf{x}\right)^{1 / 2}\left(\sum_{i=1}^{E} \int_{\omega} \rho_{\epsilon, i} m^{2}\left|\nabla q_{1}\right|^{2} d \mathbf{x}\right)^{1 / 2}+\left(r_{\gamma, 2}, q_{1}\right)_{\bar{a}} \\
& \leq \sqrt{\left|\left(r_{\gamma, 2}, p_{1}\right)_{\bar{a}}\right|} \sqrt{\frac{\rho_{+} m_{+}}{a_{-}}}\left\|q_{1}\right\|_{a}+\sqrt{\frac{a_{+}}{a_{-}}}\left\|r_{\gamma, 2}\right\|_{\bar{a}} \cdot\left\|q_{1}\right\|_{a} \\
& \leq\left(\frac{a_{+}}{a_{-}}\right)^{1 / 4} \sqrt{\frac{\rho_{+} m_{+}}{a_{-}}} \sqrt{\left\|r_{\gamma, 2}\right\|_{\bar{a}}} \sqrt{\left\|p_{1}\right\|_{a}}\left\|q_{1}\right\|_{a}+\sqrt{\frac{a_{+}}{a_{-}}}\left\|r_{\gamma, 2}\right\|_{\bar{a}} \cdot\left\|q_{1}\right\|_{a},
\end{aligned}
$$

where, we have used that $\sum_{i=1}^{E} \int_{\omega} \rho_{\epsilon, i} m^{2}\left|\nabla p_{1}\right|^{2} d \mathbf{x} \leq\left|\left(r_{\gamma, 2}, p_{1}\right)_{\bar{a}}\right|$ because of (48). Hence

$$
\left\|q_{1}\right\|_{a} \leq\left(\frac{a_{+}}{a_{-}}\right)^{1 / 4} \sqrt{\frac{\rho_{+} m_{+}}{a_{-}}} \sqrt{\left\|r_{\gamma, 2}\right\|_{\bar{a}}} \sqrt{\left\|p_{1}\right\|_{a}}+\sqrt{\frac{a_{+}}{a_{-}}}\left\|r_{\gamma, 2}\right\|_{\bar{a}},
$$

and using (49), we conclude that for $q_{1}$ :

$$
\left\|q_{1}\right\|_{a} \leq \sqrt{\frac{a_{+}}{a_{-}}}\left(c_{\omega} \sqrt{\frac{\rho_{+} m_{+}}{\alpha a_{-}}}+1\right)\left\|r_{\gamma, 2}\right\|_{\bar{a}}
$$

Analogously, by taking $\varphi=p_{2}$ in (45) we get

$$
\left\|p_{2}\right\|_{a} \leq \frac{c_{\omega}}{\alpha}\left\|q_{2}\right\|_{L^{2}(\partial \omega)}+\sqrt{\frac{a_{+}}{a_{-}}}\left\|r_{\gamma, 1}\right\|_{\bar{a}}
$$

Choosing $\varphi=q_{2}$ in (45) and $\psi=p_{2}$ in (46) and subtracting, we obtain

$$
\frac{1}{\alpha} \int_{\omega} q_{2}^{2} d \sigma+\sum_{i=1}^{E} \int_{\omega} \rho_{\epsilon, i}\left(m \nabla p_{2}\right)^{2} d \mathbf{x}=\left(r_{\gamma, 1}, q_{2}\right)_{\bar{a}},
$$


and hence

$$
\begin{aligned}
\left\|q_{2}\right\|_{L^{2}(\partial \omega)}^{2} & \leq \alpha\left\|r_{\gamma, 1}\right\|_{\bar{a}} \sqrt{\frac{a_{+}}{a_{-}}}\left\|q_{2}\right\|_{a}, \\
\sum_{i=1}^{E} \int_{\omega} \rho_{\epsilon, i}\left(m \nabla p_{2}\right)^{2} d \mathbf{x} & \leq\left(r_{\gamma, 1}, q_{2}\right)_{\bar{a}},
\end{aligned}
$$

On the other hand, taking $\psi=q_{2}$ in (46), we have

$$
\begin{aligned}
\left\|q_{2}\right\|_{a}^{2} & =\sum_{i=1}^{E} \int_{\omega} \rho_{\epsilon, i}\left(m \nabla p_{2}\right)\left(m \nabla q_{2}\right) d \mathbf{x} \leq\left(\sum_{i=1}^{E} \int_{\omega} \rho_{\epsilon, i} m^{2}\left|\nabla p_{2}\right|^{2} d \mathbf{x}\right)^{1 / 2}\left(\sum_{i=1}^{E} \int_{\omega} \varphi_{\epsilon, i} m^{2}\left|\nabla q_{2}\right|^{2} d \mathbf{x}\right)^{1 / 2} \\
& \leq \sqrt{\left|\left(r_{\gamma, 1}, q_{2}\right)_{\bar{a}}\right|} \sqrt{\frac{\rho_{+} m_{+}}{a_{-}}}\left\|q_{2}\right\|_{a}
\end{aligned}
$$

where we have used (53). Hence, we obtain the following bound for $q_{2}$

$$
\left\|q_{2}\right\|_{a} \leq \sqrt{\frac{a_{+}}{a_{-}}} \frac{\rho_{+} m_{+}}{a_{-}}\left\|r_{\gamma, 1}\right\|_{\bar{a}} .
$$

Now, from (51) and (52), we have

$$
\left\|p_{2}\right\|_{a} \leq \frac{c_{\omega}}{\alpha} \sqrt{\alpha}\left(\frac{a_{+}}{a_{-}}\right)^{1 / 4} \sqrt{\left\|r_{\gamma, 1}\right\|_{\bar{a}}} \sqrt{\left\|q_{2}\right\|_{a}}+\sqrt{\frac{a_{+}}{a_{-}}}\left\|r_{\gamma, 1}\right\|_{\bar{a}},
$$

and using (54) we obtain for $p_{2}$ :

$$
\left\|p_{2}\right\|_{a} \leq \sqrt{\frac{a_{+}}{a_{-}}}\left(\frac{c_{\omega}}{\sqrt{\alpha}} \sqrt{\frac{\rho_{+} m_{+}}{a_{-}}}+1\right)\left\|r_{\gamma, 1}\right\|_{\bar{a}} .
$$

Finally, combining (49), (50), (54), and (55), we obtain the desired result.

Remark 3.1 (Approximation of $C_{1}$ and $C_{2}$ ). In order to get an a posteriori error bound, we need to estimate the constants $C_{1}$ and $C_{2}$, and therefore $c_{\omega}$ in (22). It is easy to show [15] that $c_{\omega}$ is the inverse of the square root of the least positive eigenvalue associated to the problem

$$
\begin{array}{rlr}
\nabla \cdot a \nabla u & =0 & \text { in } \Omega, \\
\nabla u \cdot \mathbf{n} & =\lambda u & \text { on } \partial \Omega .
\end{array}
$$

Remark 3.2 (Estimation based on the inf-sup). Using Proposition 2.1, it is possible to obtain a similar result to (41) (see $[14,16])$ :

$$
\left\|\left(e_{p}(\gamma), e_{q}(\gamma)\right)\right\|_{a} \leq \frac{1}{\beta_{\mathcal{A}(\gamma)}} \sqrt{\frac{a_{+}}{a_{-}}}\left(\left\|r_{\gamma, 1}\right\|_{\bar{a}}+\left\|r_{\gamma, 2}\right\|_{\bar{a}}\right) .
$$

We reject this estimation, because the worse term, $\frac{c_{\omega}^{2}}{\alpha}$, affects both the residuals $r_{\gamma, 1}$ and $r_{\gamma, 2}$.

Remark 3.3 (Estimation at continuous level). Note that the estimation in Proposition 3.1 is also valid for the error $\left(p-p_{h}, q-q_{h}\right)$, where $(p, q)$ is the solution of problem (19) and $\left(p_{h}, q_{h}\right)$ is the FE approximation (29).

In view of Proposition 3.1, we introduce the estimator and its corresponding effectivity index as:

$$
\Delta_{N}(\gamma)=C_{1}\left\|r_{\gamma, 1}\right\|_{\bar{a}}+C_{2}\left\|r_{\gamma, 2}\right\|_{\bar{a}}, \quad \eta_{N}(\gamma)=\frac{\Delta_{N}(\gamma)}{\left\|\left(e_{p}(\gamma), e_{q}(\gamma)\right)\right\|_{a}} .
$$

We end this section with the folowing result: 
Proposition 3.2. For any $N>0$ and $\gamma \in \mathcal{D}$, the effectivity index satisfies

$$
1 \leq \eta_{N}(\gamma) \leq \max \left\{C_{1}, C_{2}\right\} C_{\mathcal{A}} \sqrt{\frac{a_{+}}{2 a_{-}}},
$$

where $C_{\mathcal{A}}$ is the continuity constant of $\mathcal{A}_{\gamma}(\cdot, \cdot)$ and $C_{1}$ and $C_{2}$ are given by (42).

Proof. The lower bound is a simple consequence of (57). We next resort to (39) and (40) to obtain:

$$
\begin{aligned}
\Delta_{N}(\gamma) & \leq \max \left\{C_{1}, C_{2}\right\}\left(\left\|r_{\gamma, 1}\right\|_{\bar{a}}+\left\|r_{\gamma, 2}\right\|_{\bar{a}}\right) \leq \frac{\max \left\{C_{1}, C_{2}\right\}}{\sqrt{2}}\left\|\left(r_{\gamma, 1}, r_{\gamma, 2}\right)\right\|_{\bar{a}} \\
& =\frac{\max \left\{C_{1}, C_{2}\right\}}{\sqrt{2}} \sup _{\left(\varphi_{h}, \psi_{h}\right) \in \mathbb{V}^{\circ}{ }_{h} \times \mathbb{V}^{\circ}{ }_{h}} \frac{\mathcal{A}_{\gamma}\left(\left(e_{p}(\gamma), e_{q}(\gamma)\right),\left(\varphi_{h}, \psi_{h}\right)\right)}{\left\|\left(\varphi_{h}, \psi_{h}\right)\right\|_{\bar{a}}} \leq \max \left\{C_{1}, C_{2}\right\} \sqrt{\frac{a_{+}}{2 a_{-}}} C_{\mathcal{A}}\left\|\left(e_{p}(\gamma), e_{q}(\gamma)\right),\right\|_{a}
\end{aligned}
$$

which gives the upper bound in (58) and concludes the proof.

\section{Numerical experiments}

In this section, we present two numerical examples that illustrate the performance of our reduced basis scheme and confirm its efficiency.

In order to implement the mean zero condition for the traces of functions in $\mathbb{V}^{\circ}$, we introduce Lagrange multipliers. That is, instead of (19), we consider the equivalent problem: find $\left(p, q, \lambda_{1}, \lambda_{2}\right) \in \mathbb{V} \times \mathbb{V} \times \mathbb{R} \times \mathbb{R}$ such that

$$
\begin{aligned}
& \mathcal{A}_{\gamma}((p, q),(\varphi, \psi))+\lambda_{1} \int_{\partial \omega} \varphi+\lambda_{2} \int_{\partial \omega} \psi=\mathcal{F}_{\gamma}((\varphi, \psi)), \quad \forall(\varphi, \psi) \in V \times V \\
& \mu_{1} \int_{\partial \omega} p+\mu_{2} \int_{\partial \omega} q=0 \quad \forall\left(\mu_{1}, \mu_{2}\right) \in \mathbb{R} \times \mathbb{R}
\end{aligned}
$$

In the next theorem we establish the equivalence between (19) and (59).

Proposition 4.1. Let $(p, q) \in \mathbb{V}^{\circ} \times \mathbb{V}^{\circ}$ be the solution of (19). Then, $(p, q, 0,0)$ is solution of (59). Conversely, let $\left(p, q, \lambda_{1}, \lambda_{2}\right) \in \mathbb{V} \times \mathbb{V} \times \mathbb{R} \times \mathbb{R}$ be the solution of (59). Then $\lambda_{1}=\lambda_{2}=0$ and $(p, q)$ is the solution of (19). In particular, (59) has a unique solution.

Proof. Let $(p, q) \in \mathbb{V}^{\circ} \times \mathbb{V}^{\circ}$ be the solution of (19), and $(\varphi, \psi) \in \mathbb{V} \times \mathbb{V}$. We introduce $c_{\varphi}=\frac{1}{|\partial \omega|} \int_{\partial \omega} \varphi$ and $c_{\psi}=\frac{1}{|\partial \omega|} \int_{\partial \omega} \psi$, and note that $\widetilde{\varphi}:=\varphi-c_{\varphi} \in \mathbb{V}^{\circ}$ and $\widetilde{\psi}:=\psi-c_{\psi} \in \mathbb{V}^{\circ}$. From the definitions of $\mathcal{A}_{\gamma}(\cdot, \cdot)$ and $\mathcal{F}_{\gamma}(\cdot)$, and since $p \in \mathbb{V}^{\circ}$ (ie. $\left.\int_{\partial \omega} p=0\right)$ we have

$$
\begin{aligned}
\mathcal{A}_{\gamma}((p, q),(\varphi, \psi)) & =\mathcal{A}_{\gamma}((p, q),(\widetilde{\varphi}, \widetilde{\psi}))+\frac{1}{\alpha} c_{\varphi} \int_{\partial \omega} q \\
& =\mathcal{A}_{\gamma}((p, q),(\widetilde{\varphi}, \widetilde{\psi}))=\mathcal{F}_{\gamma}((\widetilde{\varphi}, \widetilde{\psi}))=\mathcal{F}_{\gamma}((\varphi, \psi)),
\end{aligned}
$$

where in the third identity we use that $(p, q)$ is the solution of (19). This implies that $(p, q, 0,0)$ is the solution of (59). On the other hand, let $\left(p, q, \lambda_{1}, \lambda_{2}\right) \in \mathbb{V} \times \mathbb{V} \times \mathbb{R} \times \mathbb{R}$ be the solution of (59). Then, taking $(1,0) \in \mathbb{R} \times \mathbb{R}$ in the second equation of (59) we get $\int_{\partial \omega} p=0$ and therefore $p \in \mathbb{V}^{\circ}$. Using the same argument with the pair $(0,1)$ we also obtain $q \in \mathbb{V}^{\circ}$. Now, choosing $(\varphi, \phi)=(1,0)$ in the first equation of $(59)$ we have

$$
0=\mathcal{F}_{\gamma}((1,0))=\mathcal{A}_{\gamma}((p, q),(1,0))=\frac{1}{\alpha} \int_{\partial \omega} p+\lambda_{1} \int_{\partial \omega} 1,
$$

that implies $\lambda_{1}=0$. The same computation with $(\varphi, \phi)=(0,1)$ gives $\lambda_{2}=0$. Finally, since $\mathbb{V}^{\circ} \subset \mathbb{V}$ we conclude that $(p, q)$ is the solution of (19). 
The numerical experiments were performed with the finite element toolbox FreeFEM[17] and the MATLAB environment. The generation of the mesh (adapted to the orography of the terrain) and the assemblage of the matrices were done with FreeFEM. The solution of the linear system was computing with MATLAB (backslash operator). Given the features of system (59), MATLAB chooses a LU solver (UMFPACK routines). An efficient strategy to solve this type of problem could also be implemented[18]. The offline/online procedures were developed on MATLAB. Parallelism was exploited to speed up the evaluation of the a posteriori error estimate during the greedy algorithm. The simulations were performed on a Dell Precision T7610 workstation, equipped with 2 Intel Xeon E52670 v2 processors (10 cores each one) and 64 GB RAM. From [19] we choose the parameters $\left\{d_{0}, d_{1}, d_{2}\right\}$ in the range: $[0.8,1] \times[-0.4,-0.2] \times[0.025,0.05]$. The parameter independent norm is defined for values $\left\{\bar{d}_{0}=0.9, \bar{d}_{1}=-0.3, \bar{d}_{2}=0.015\right\}$.

\subsection{Example 1: A toy problem}

We first deal with a simple example where we analyze the effect of orography and roughness on the wind. We consider a domain $\omega=6 \times 6(\mathrm{~km})$ with two hills and composed of two subdomains with different roughness (see Figure 4.1). We also assume $\delta=0.5(\mathrm{~km})$ and four observations points at coordinates $(1,1),(1,5),(5,1)$ and $(5,5)$. Then, our problem admits an affine decomposition with $J_{a}=10$ and $J_{l}=32$ (compare with section 3.1) and the number of parameters is $3+2 \times 4=11$ (roughness adjustment and two components of velocity at each point).

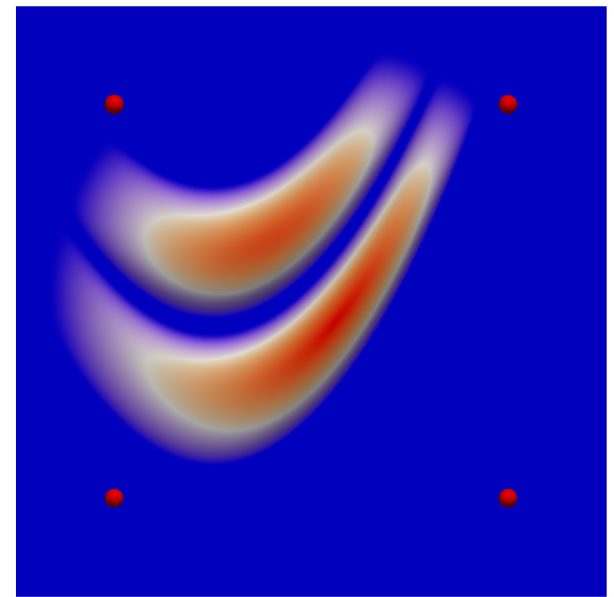

Elevation $(\mathrm{km})$ and Observation Points

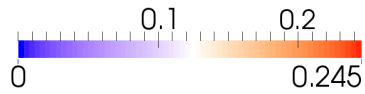

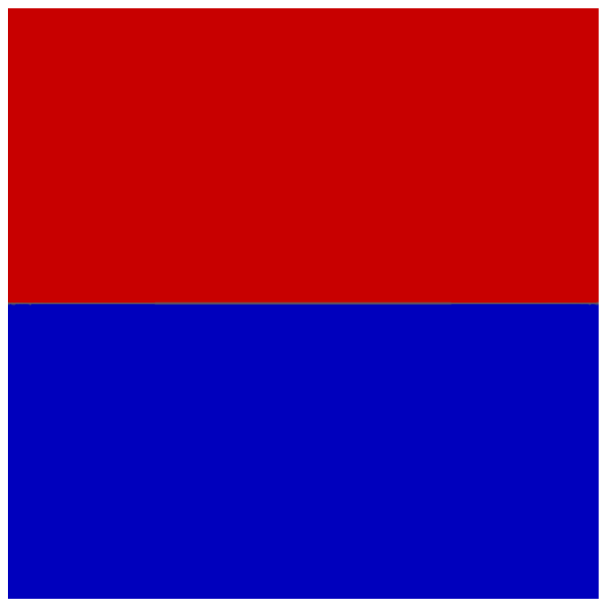

$\mathrm{ZO}$

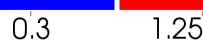

Figure 1: Example 1: Orography, observation points (left) and roughness (right).

For the FE discretization we use piecewise linear elements on a mesh $\mathcal{T}_{h}$ of 131072 triangles. The total number of degrees of freedom is 132100 , i.e. the size of the system (59).

The training set $\Xi \subset \mathcal{D}$ is chosen as follows. We discretize the range of parameters $\left\{d_{0}, d_{1}, d_{2}\right\}$ with a set of 64 equidistributed points in $[0.8,1] \times[-0.4,-0.2] \times[0.025,0.05]$. About the velocity, we consider four directions (North, South, East and West) and where its module can be active $(\|\mathbf{V}\|=10 \mathrm{~km} / \mathrm{h})$ or inactive $(\|\mathbf{V}\|=0)$ at each station. It gives a set $\Xi$ of cardinality 39936. As we mentioned in section 3.3, the computation of the a posteriori estimator requires the constants $C_{1}$ and $C_{2}$ in (57). In order to approximate them, we solve the eigenvalue problem (56) for each value of $\left\{d_{0}, d_{1}, d_{2}\right\}$ during the offline procedure. To estimate the error on the online process we use linear interpolation to get $C_{1}$ and $C_{2}$.

The greedy procedure for the construction of the RB space selects $\mathrm{N}=64$ for a tolerance $t o l=1 . e-2$. We note that the number of basis functions required to achieve this tolerance does not vary for finer discretization of the parameters space $\mathcal{D}$. In Figure 2 (left) we show the evolution of the error estimator $\Delta(\gamma)$ and of the true error between the FE and 
RB approximations during the greedy algorithm. Although the estimation is not sharp, we point out a good correlation with the true error. In Figure 2 (right) we report the effectivity index.
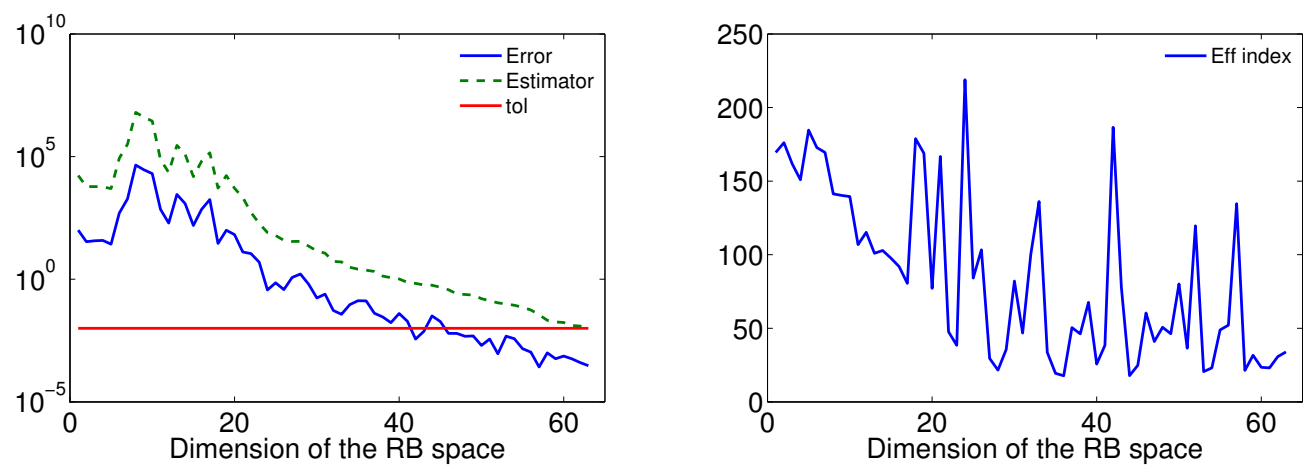

Figure 2: Example 1: Evolution of the estimator and of the true error during the greedy procedure (left) and its corresponding effectivity index.

As regards the computational performances, the time spent in the offline procedure is about 10 hours, while the online procedure (which demands the computation of the solution and the estimation of the error) only requires $0.3 \mathrm{~s}$. Let us remark that the solution of the FE problem (assemblage and solution of the linear system) needs about $12 \mathrm{~s}$. It provides a reduction factor $1: 40$.

Finally, we solve the problem for $\left\{d_{0}, d_{1}, d_{2}\right\}=\{0.9,-0.2,0.03\}$, and uniform measures of velocity given by a module $10 \mathrm{~km} / \mathrm{h}$ and eastward direction. We report details of the solution in Figures 3 and 4 . As expected, the wind field is deflected by the hills and perturbed by the roughness of the terrain. In this example, where the approximation of the constants $C_{1}$ and $C_{2}$ requires interpolation, we obtain $\Delta(\gamma) \approx 7.5 e-3$ and $\left(\left\|e_{p}(\gamma), e_{q}(\gamma)\right\|_{a} \approx 2.5 e-4\right.$, which corroborates the reliability of the RB approximation.

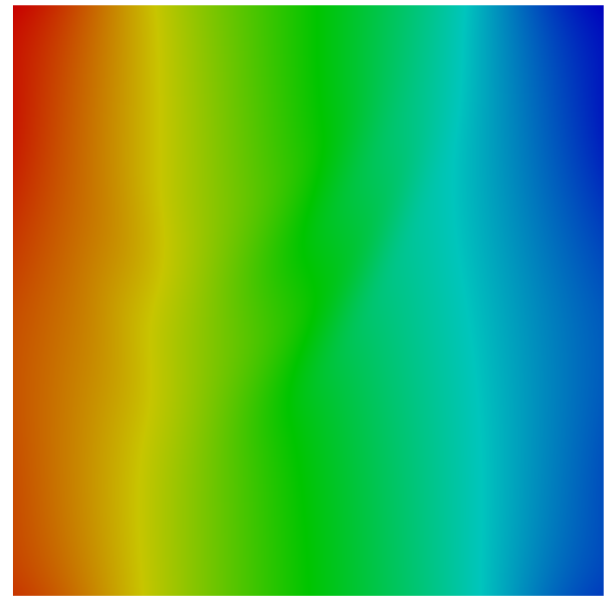

Potential

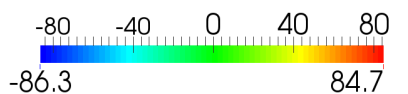

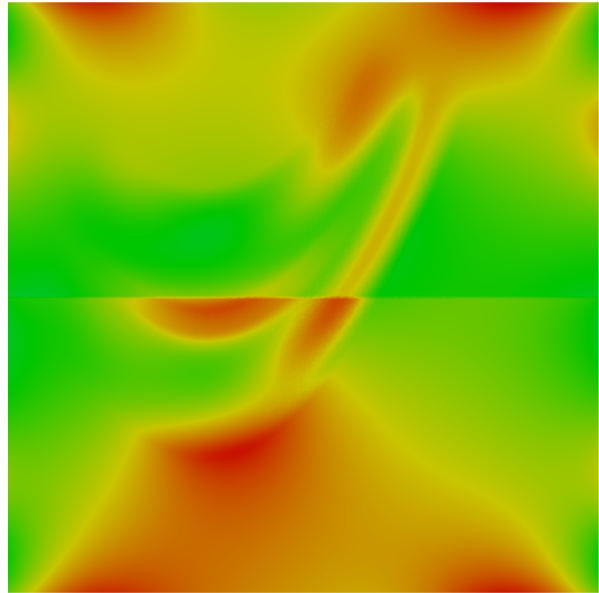

Velocity (module) $(\mathrm{km} / \mathrm{h})$

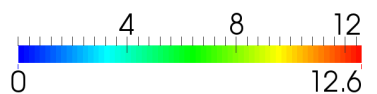

Figure 3: Example 1: Potential and module of the velocity $10 \mathrm{~m}$ above the terrain. 


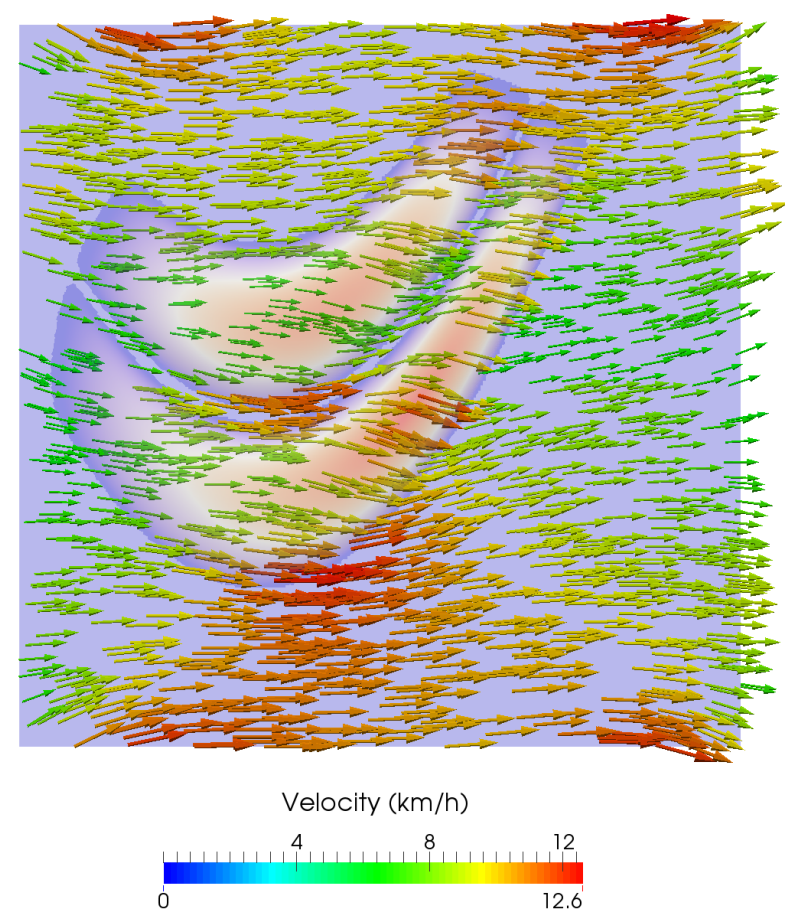

Figure 4: Example 1: Velocity field $10 \mathrm{~m}$ above the terrain.

\subsection{Example 2: A real case}

We now consider a scenario with real data. The studied domain is located at Quintero-Puchuncaví area (Chile, $\mathrm{V}$ Region). It has dimensions $12 \times 12(\mathrm{~km})$ and the upper boundary has been taken at a height $\delta=0.7(\mathrm{~km})$. There are four weather stations in the area (Quintero(1), Valle Alegre(2), La Greda(3), Sur(4)), that we use as data. The wind measures were provided by SINCA, [20]. A digital elevation map of the domain and its roughness data is used. In Figure 5 we show the orography of the terrain, the weather station location and its roughness map.

As in the previous case, we obtain an affine decomposition with $J_{a}=10$ and $J_{l}=32$. The training set $\Xi \in \mathcal{D}$ is chosen as in example 1, i.e. $\# \Xi=39936$. The FE discretization uses piecewise linear elements on a mesh $\mathcal{T}_{h}$ of 205474 triangles, that provides 206678 degrees of freedom.

The greedy procedure for the construction of the RB space selects $\mathrm{N}=181$ for a tolerance $t o l=1 . e-2$. We report in Figure 6 (left) the evolution of the estimator $\Delta(\gamma)$ and of the true error during the greedy algorithm. Again, we observe a good correlation between estimator and error.

As regards the computational performances, the time spent in the offline procedure to build the RB spaces is about 4 days in our system. However, the online procedure only requires $1.02 \mathrm{~s}$. On the other hand the FE problem needs about $46 \mathrm{~s}$. to provide a solution. It provides a reduction factor 1:45.

We conclude with the wind field simulation corresponding to the measures shown in Table 1. In this example we take $\left\{d_{0}, d_{1}, d_{2}\right\}=\{0.95,-0.23,0.045\}$. We show details of the solution in Figures 7 and 8 . The online procedure required $4 \mathrm{~s}$. to solve the problem and provided $\Delta_{N}(\gamma)=7.3 . e-3$.

\section{Acknowledgments}

The first and third authors were partially supported by Grant CGL2011-29396-C03-02, Ministerio de Economía y Competitividad (Spain). The second author was partially supported by FONDECYT N1140392, CONICYT grant ACT-1106, and Basal CMM U. de Chile. 


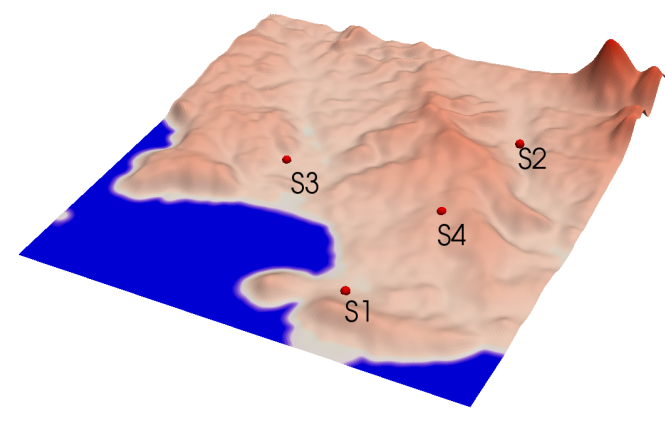

Elevation (km) and Observation Points (red)

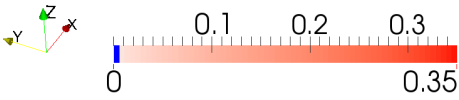

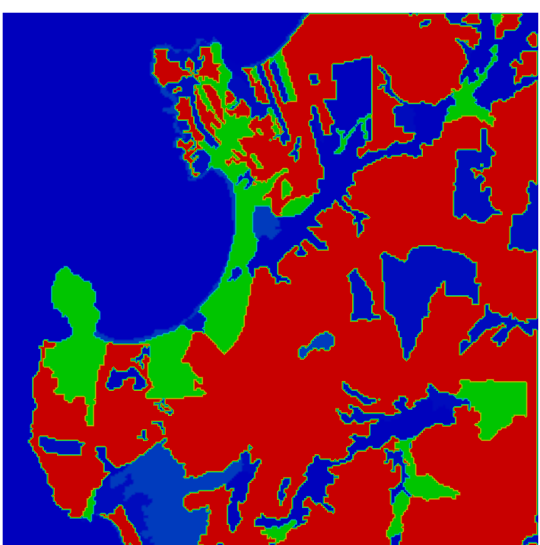

$\mathrm{ZO}$

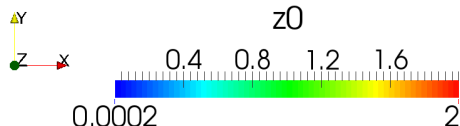

Figure 5: Example 2: Orography, observation points (left) and roughness map (right).

\begin{tabular}{lll}
\hline Weather Station & $\begin{array}{l}\text { Module } \\
(\mathrm{Km} / \mathrm{h})\end{array}$ & $\begin{array}{l}\text { Direction } \\
\text { (clockwise from North) }\end{array}$ \\
\hline \hline 1 & 11.04 & 301.60 \\
\hline 2 & 6.86 & 259.80 \\
\hline 3 & 13.30 & 295.60 \\
\hline 4 & 11.40 & 297.70 \\
\hline
\end{tabular}

Table 1: Example 2: Wind measures at the weather stations.

\section{References}

[1] M. Asensio, L. Ferragut, J. Simon, A convection model for fire spread simulation, Applied Mathematics Letters 18 (6) (2005) 673 - 677, special issue on the occasion of \{MEGA\} 2003MEGA 2003. doi:http://dx.doi.org/10.1016/j.aml.2004.04.011.

URL http://www.sciencedirect.com/science/article/pii/S0893965904003787

[2] L. Ferragut, M. I. Asensio, J. Simon, High definition local adjustment model of $3 \mathrm{~d}$ wind fields performing only $2 \mathrm{~d}$ computations, International Journal for Numerical Methods in Biomedical Engineering 27 (4) (2011) 510-523. doi:10.1002/cnm.1314.

URL http://dx.doi.org/10.1002/cnm.1314

[3] J. L. Lions, Contrôle Optimal de Systèmes Gouvernés par des Équations aux Dérivés Partielles, Springer series in computational mathematics, Dunod/Gauthier-Villars, Paris, 1968

[4] L. Ferragut, R. Montenegro, G. Montero, E. Rodríguez, M. Asensio, J. Escobar, Comparison between 2.5-d and 3-d realistic models for wind field adjustment, Journal of Wind Engineering and Industrial Aerodynamics 98 (1011) (2010) 548 - 558. doi:http://dx.doi.org/10.1016/j.jweia.2010.04.004.

URL http://www.sciencedirect.com/science/article/pii/S0167610510000346

[5] A. Quarteroni, A. Manzoni, F. Negri, Reduced basis methods for partial differential equations, Vol. 92 of Unitext, Springer, Cham, 2016, an introduction, La Matematica per il 3+2.

[6] G. Rozza, Reduced basis approximation and error bounds for potential flows in parametrized geometries, Communications in Computational Physics 9 (1) (2011) 1-48, preprint as MATHICSE report 11.2010. doi:10.4208/cicp.100310.260710a.

URL http: //augustine.mit.edu/

[7] P. Pacciarini, G. Rozza, Stabilized reduced basis method for parametrized advection-diffusion PDEs, Computer Methods in Applied Mechanics and Engineering 274 (2014) 1 - 18. doi:http://dx.doi.org/10.1016/j.cma.2014.02.005. URL http://www.sciencedirect.com/science/article/pii/S0045782514000565

[8] A. Manzoni, F. Salmoiraghi, L. Heltai, Reduced basis isogeometric methods (rb-iga) for the real-time simulation of potential flows about parametrized $\{\mathrm{NACA}\}$ airfoils, Computer Methods in Applied Mechanics and Engineering 284 (2015) 1147 - 1180, isogeometric Analysis 

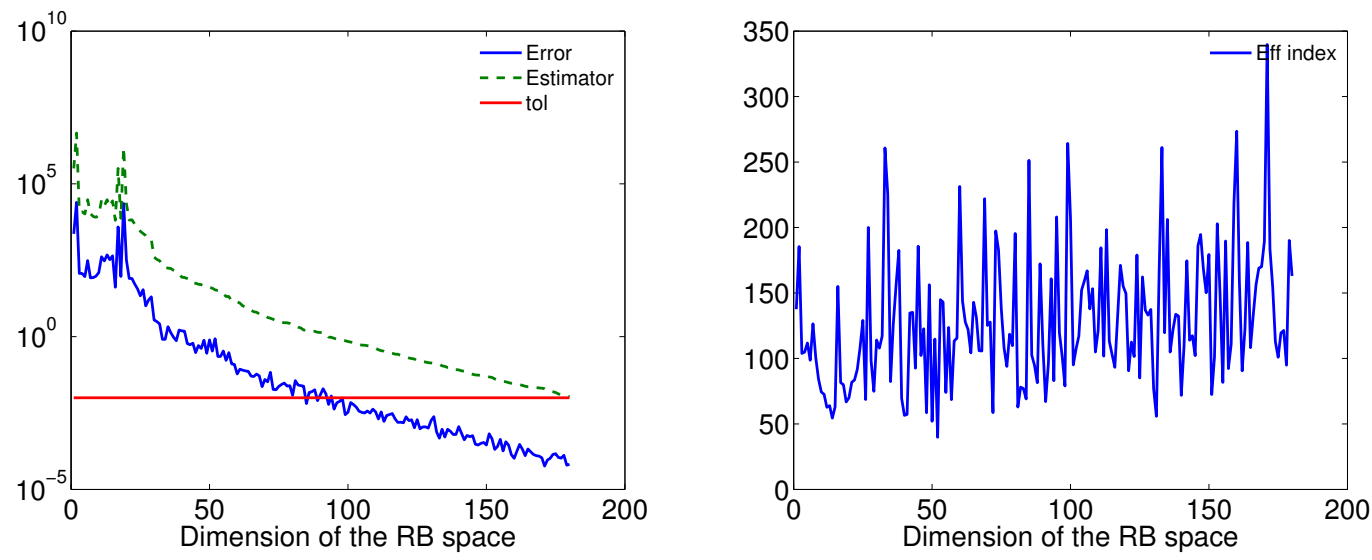

Figure 6: Example 2: Evolution of the estimator and of the true error during the greedy procedure (left) and its corresponding effectivity index.

Special Issue. doi:http://dx.doi.org/10.1016/j.cma.2014.11.037.

URL http: //www.sciencedirect.com/science/article/pii/S0045782514004708

[9] K. Hoang, P. Kerfriden, S. Bordas, A fast, certified and "tuning free" two-field reduced basis method for the metamodelling of affinely-parametrised elasticity problems, Computer Methods in Applied Mechanics and Engineering 298 (2016) 121 - 158. doi:http://dx.doi.org/10.1016/j.cma.2015.08.016.

URL http://www.sciencedirect.com/science/article/pii/S0045782515002741

[10] D. Carvalho, A. Rocha, C. S. Santos, R. Pereira, Wind resource modelling in complex terrain using different mesoscale-microscale coupling techniques, Applied Energy 108 (2013) 493 - 504. doi:http://dx.doi.org/10.1016/j.apenergy.2013.03.074.

URL http://www.sciencedirect.com/science/article/pii/S0306261913002705

[11] H. Gopalan, C. Gundling, K. Brown, B. Roget, J. Sitaraman, J. D. Mirocha, W. O. Miller, A coupled mesoscale-microscale framework for wind resource estimation and farm aerodynamics, Journal of Wind Engineering and Industrial Aerodynamics 132 (2014) 13 - 26. doi:http://dx.doi.org/10.1016/j.jweia.2014.06.001.

URL http://www.sciencedirect.com/science/article/pii/S016761051400107X

[12] E. Marino, C. Lugni, C. Borri, A novel numerical strategy for the simulation of irregular nonlinear waves and their effects on the dynamic response of offshore wind turbines, Computer Methods in Applied Mechanics and Engineering 255 (2013) 275 - 288. doi:http://dx.doi.org/10.1016/j.cma.2012.12.005.

URL http://www.sciencedirect.com/science/article/pii/S0045782512003751

[13] D. Boffi, M. Fortin, F. Brezzi, Mixed finite element methods and applications, Springer series in computational mathematics, Springer, Berlin, Heidelberg, 2013.

URL http: //opac.inria.fr/record=b1135229

[14] F. Negri, G. Rozza, A. Manzoni, A. Quarteroni, Reduced basis method for parametrized elliptic optimal control problems, SIAM Journal on Scientific Computing 35 (5) (2013) A2316-A2340. arXiv:http://dx.doi.org/10.1137/120894737, doi:10.1137/120894737. URL http://dx.doi.org/10.1137/120894737

[15] A. I. Nazarov, S. I. Repin, Exact constants in poincarè type inequalities for functions with zero mean boundary traces, Mathematical Methods in the Applied Sciences 38 (15) (2015) 3195-3207. doi:10.1002/mma.3290. URL http://dx.doi.org/10.1002/mma.3290

[16] F. Negri, A. Manzoni, G. Rozza, Reduced basis approximation of parametrized optimal flow control problems for the stokes equations, Computers \& Mathematics with Applications 69 (4) (2015) 319 - 336. doi:http://dx.doi.org/10.1016/j.camwa.2014.12.010.

URL http://www.sciencedirect.com/science/article/pii/s0898122114006075

[17] F. Hecht, New development in freefem++, J. Numer. Math. 20 (3-4) (2012) 251-265.

[18] M. Benzi, L. Ferragut, M. Pennacchio, V. Simoncini, Solution of linear systems from an optimal control problem arising in wind simulation, Numerical Linear Algebra with Applications 17 (6) (2010) 895-915. doi:10.1002/nla.679. URL http: //dx.doi.org/10.1002/nla.679

[19] L. Ferragut, R. Montenegro, G. Montero, E. Rodríguez, M. Asensio, J. Escobar, Comparison between 2.5-d and 3-d realistic models for wind field adjustment, Journal of Wind Engineering and Industrial Aerodynamics 98 (1011) (2010) 548 - 558. doi:http://dx.doi.org/10.1016/j.jweia.2010.04.004. URL http: //www. sciencedirect.com/science/article/pii/S0167610510000346

[20] SINCA, Ministerio de Medio Ambiente (Gobierno de Chile), Sistema de Información Nacional de Calidad del Aire (2016). URL http://http://sinca.mma.gob.cl 

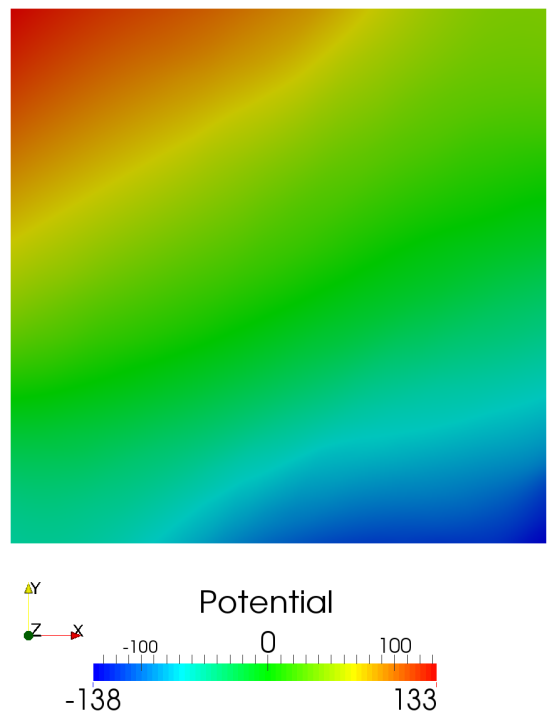
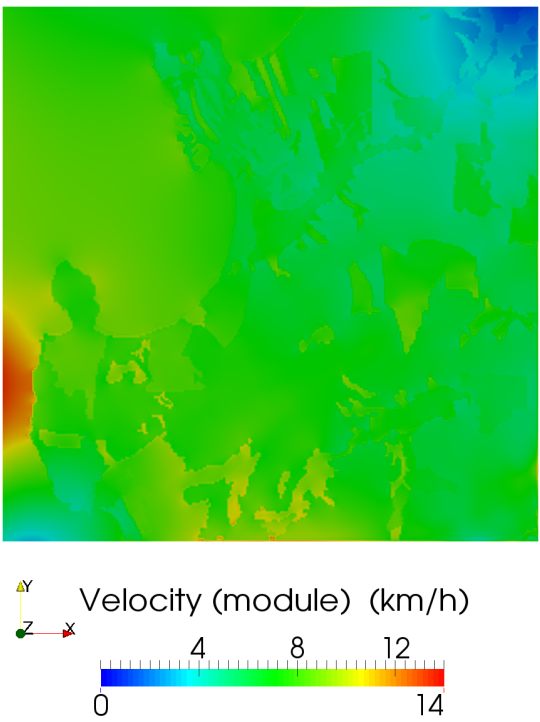

Figure 7: Example 2: Potential and module of velocity $10 \mathrm{~m}$ above terrain.

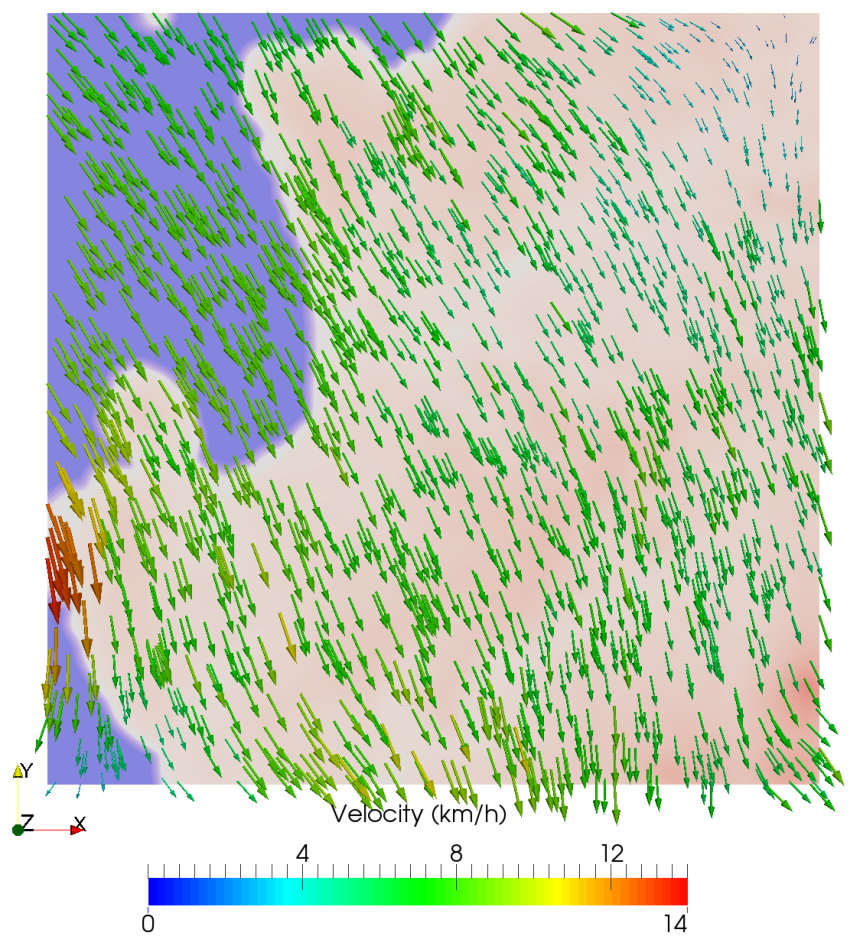

Figure 8: Example 2: Velocity field $10 \mathrm{~m}$ above terrain. 